\title{
Itch expression by Treg cells controls Th2 inflammatory responses
}

\author{
Hyung-seung Jin, Yoon Park, Chris Elly, and Yun-Cai Liu \\ Division of Cell Biology, La Jolla Institute for Allergy and Immunology, La Jolla, California, USA.
}

\begin{abstract}
Regulatory $\mathrm{T}$ (Treg) cells maintain immune homeostasis by limiting autoimmune and inflammatory responses. Treg differentiation, maintenance, and function are controlled by the transcription factor Foxp3. However, the exact molecular mechanisms underlying Treg cell regulation remain elusive. Here, we show that Treg cell-specific ablation of the $\mathrm{E} 3$ ubiquitin ligase Itch in mice caused massive multiorgan lymphocyte infiltration and skin lesions, chronic $T$ cell activation, and the development of severe antigen-induced airway inflammation. Surprisingly, Foxp3 expression, homeostasis, and the in vitro and in vivo suppressive capability of Treg cells were not affected by Itch deficiency. We found that the expression of Th2 cytokines by Treg cells was increased in the absence of Itch. Fate mapping revealed that a fraction of Treg cells lost Foxp3 expression independently of Itch. However, Th2 cytokines were excessively augmented in Itch-/-Foxp3-negative "ex-Treg" cells without altering the percentage of conversion. Targeted knockdown of Th2 transcriptional regulators in $\mathrm{Itch}^{-/-}$Treg cells prevented Th2 cytokine production. The present study unveils a mechanism of Treg cell acquisition of Th2-like properties that is independent of Foxp3 function and Treg cell stability.
\end{abstract}

\section{Introduction}

Regulatory $\mathrm{T}$ (Treg) cells constitute a unique subset of $\mathrm{CD}^{+} \mathrm{T}$ cells that control autoimmunity and inflammatory pathology to maintain immune homeostasis via actively suppressing selfreactive T cells $(1,2)$. Foxp3, an X chromosome-encoded forkhead domain-containing transcription factor, is the master regulator responsible for the differentiation, maintenance, and function of Treg cells $(3,4)$. Its mutation or deficiency is associated with excessive autoimmune diseases, as revealed in Scurfy mutant mice and human patients with immune dysregulation, polyendocrinopathy, enteropathy, and X-linked (IPEX) syndromes $(5,6)$. Treg cells are generated in the thymus and migrate into the periphery to become naturally occurring Treg (nTreg) cells (7). In addition, peripheral naive $\mathrm{CD}^{+} \mathrm{T}$ cells can also be induced into Foxp $3^{+}$Treg (iTreg) cells either by transforming growth factor $\beta$ (TGF- $\beta$ ) in vitro (8), or by high-dose antigen administration in vivo (9). Unlike nTreg cells that possess stable genetic markers, iTreg cells may be only partially epigenetically modified (10). Treg cells exert immunosuppressive activity via multiple mechanisms including cell-cell contacts or secretion of soluble factors such as IL-10 and TGF- $\beta$, or by influencing the stimulating APCs $(11,12)$.

A growing body of evidence has indicated that Treg cells are able to suppress different types of T helper (Th) cell-mediated immune responses through the acquisition of specific $\mathrm{T}$ effector transcriptional programs and that there is functional specialization depending on the type of immune response (13). For example, Treg lineage-specific suppression of Th1, Th2, and Th17 cells was demonstrated through specific transcription factors expressed in Treg cells including Tbx21 (T-bet), IRF4, and STAT3, respectively (14-16). Additional studies have demonstrated that Treg cells display plasticity and can be converted into various effector Th cells in response to lymphopenia or inflammatory cytokine signals (17-20). However, the issue of Treg cell plasticity was largely disputed by a thorough investigation using different model systems

Conflict of interest: The authors have declared that no conflict of interest exists. Citation for this article: J Clin Invest. 2013;123(11):4923-4934. doi:10.1172/JCI69355.
(21). A more recent work by Miyao et al. (22) attempted to provide an explanation for the conflicting observations by demonstrating that a minor population of uncommitted Foxp $3^{+} \mathrm{T}$ cells undergoes phenotypic changes to acquire inflammatory effector functions under certain conditions. However, the molecular regulatory mechanisms underlying Treg plasticity or maintenance and their importance in immune responses remain to be elucidated.

The posttranslational modification mediated by ubiquitin conjugation is one of the key regulatory mechanisms that control innate or adaptive immune responses $(23,24)$. Ubiquitin conjugation to a protein substrate involves a cascade of enzymatic reactions including IL-4, E2, and E3 enzymes (25). Itch is a HECT-type E3 ubiquitin ligase involved in the regulation of immune responses, as Itchdeficient mice develop a skin-scratching phenotype and immunological disorders manifested by lymphadenopathy, splenomegaly, and inflammation in the lungs and digestive tract (26). The inflammatory phenotype is associated with hyperactivation of Itch-deficient $\mathrm{CD} 4^{+} \mathrm{T}$ cells that produce Th2 cytokines (27). Our previous study using systemic Itch knockout mice suggested that Itch is involved in the generation of iTreg cells by targeting ubiquitination of the transcription factor TIEG1 (TGF- $\beta$-inducible early gene 1 product) (28). However, another study demonstrated that overproduction of IL-4 renders Itch ${ }^{-/-}$T cells defective in differentiating into iTreg cells (29). Given the dominant role of committed nTreg cells in immune tolerance induction, these previous studies raise the critical question of whether Itch plays an intrinsic role in controlling the development and function of nTreg cells. To address this issue, we generated Itch-floxed mice carrying the Treg cell-specific Foxp3-YFP-Cre gene to conditionally delete Itch in Treg cells. The mutant mice spontaneously developed skin disorder and massive multiorgan inflammation, accompanied by the hyperproduction of Th2-dependent Igs. Unlike the previous in vitro studies (28, 29), Treg cell-restricted Itch deficiency did not affect homeostasis of, or Foxp3 expression in, these cells. Furthermore, Itch-deficient Treg cells exhibited intact suppressive activity both in vitro and in vivo. Interestingly, Itch deficiency in Treg cells resulted in the acquisition of the ability to produce Th2 inflammatory cytokines, which 
were further augmented in Itch-/- Foxp3-negative "ex-Treg" cells. The present study indicates that Treg cell-restricted Itch expression plays an intrinsic role in inhibiting the conversion of Treg cells into pathogenic Th2 effector cells, which is independent of Foxp3 induction, Treg cell suppressive function, and stability.

\section{Results}

Ablation of Itch in Treg cells results in an inflammatory disorder. To examine the Treg cell-specific function of Itch, we used mice in which the Itch gene was flanked by loxP sites (Supplemental Figure 1; supplemental material available online with this article; doi:10.1172/JCI69355DS1), and then crossed these mice with Foxp $3^{\text {Cre }}$ mice expressing the yellow fluorescent protein-Cre (YFPCre) recombinase fusion protein under the control of the Foxp3 promoter (30). We confirmed efficient and specific deletion of Itch in sorted $\mathrm{YFP}^{+}$Treg cells, but not in $\mathrm{YFP}^{-}$conventional $\mathrm{T}$ cells, by immunoblot analysis of Itch (Figure 1A). Itch $h^{f l f l}$ Foxp $3^{\mathrm{Cre}}$ mice appeared normal at birth. However, starting from 6 weeks of age, $I_{c h}{ }^{f l / f l} F_{0 x p} 3^{C r e}$ mice exhibited a lymphoproliferative disorder, pulmonary inflammation, skin lesions (Figure 1B), body weight loss (Figure 1C), higher mortality (Figure 1D), and a larger size and cellularity of peripheral lymphoid organs (Figure 1, E and F). In addition, histological analysis of $I t c f^{f / f l} \mathrm{Foxp} 3^{\mathrm{Cre}}$ mice showed massive infiltration in the lungs, stomach, skin, and liver (Figure 1G). The degree of lung and skin inflammation was scored on H\&Estained sections, as described in Methods (Figure 1H). A large number of leukocytes were present in the splenic red pulp of the mice (Figure 1G), however, the kidney and colon did not exhibit noticeable lesions in $\mathrm{Itch}^{f / f l} \mathrm{Foxp} 3^{\mathrm{Cre}}$ mice (Supplemental Figure 2). As Itch has been implicated in Th2-mediated immune diseases, we used an antigen-induced airway inflammation model of asthma. Young (4- to 5-week-old) $\mathrm{Itch}^{+/+}$Foxp $3^{\mathrm{Cre}}$ or $\mathrm{Itch}^{f / f f l} \mathrm{Foxp} 3^{\mathrm{Cre}}$ mice were primed with OVA antigen in alum and subsequently challenged with aerosolized OVA. Twenty-four hours after the last OVA challenge, the mice were killed to measure several parameters that are indicative of airway inflammation. Itch fl/fl $F o x p 3^{C r e}$ mice exhibited more severe lung inflammation as evidenced by the increased infiltration of inflammatory cells in the BAL fluid (Figure 1I) and by histological analysis of lung tissue (Supplemental Figure 3A). Consistent with these findings, we observed a dramatic increase in OVA-specific IgE and Th2 cytokines, including IL-4, IL-5, and IL-13, in the BAL fluid (Figure 1J and Supplemental Figure 3B). To determine whether the inflammatory disease observed in Itch $f^{f / f l}$ Foxp $3^{\text {Cre }}$ mice is associated with augmented Th17 responses, we compared the susceptibility of Itch ${ }^{+/+} \mathrm{Foxp} 3^{\mathrm{Cre}}$ or $\mathrm{Itch} \mathrm{fl}^{\mathrm{l} f \mathrm{fl}} \mathrm{Foxp} 3^{\mathrm{Cre}}$ mice to experimental autoimmune encephalomyelitis (EAE) induction. The onset, severity, and duration of EAE were indistinguishable in these two groups of mice (Supplemental Figure 4A). We also found by histological analysis that there were no differences in infiltration of mononuclear cells into the spinal cord between Itch ${ }^{+/+} F_{0 x p} 3^{\mathrm{Cre}}$ and $\mathrm{Itch}^{f l / f l} \mathrm{Foxp} 3^{\mathrm{Cre}}$ mice (Supplemental Figure 4B). Collectively, Treg cell-specific Itch deletion led to chronic inflammation in multiple organs and upregulated airway inflammation upon antigen challenge, suggesting the possible contribution of Itch-deficient Treg cell to the regulation of Th2 responses.

Itch deficiency in Treg cells resulted in dysregulated Th2 responses. We next examined $\mathrm{T}$ cell development and activation in 6- to 8-weekold Itch $f^{f / f l} \mathrm{Foxp} 3^{\mathrm{Cre}}$ mice. There was no significant difference in the development of $\mathrm{CD}^{+}, \mathrm{CD}^{+}$, or $\mathrm{CD}^{+} \mathrm{CD}^{+}$thymocytes in $\mathrm{Itch}^{+/+}$ Foxp $3^{\mathrm{Cre}}$ and Itch ${ }^{f / f l} \mathrm{Foxp} 3^{\mathrm{Cre}}$ mice (Supplemental Figure 5A). The proportion of $\mathrm{CD}^{+} \mathrm{T}$ cells was slightly decreased in the spleens of $\mathrm{Itch}^{f / f l} \mathrm{Foxp} 3^{\mathrm{Cre}}$ mice compared with that in their wild-type littermates (Figure 2A, upper panel), probably due to a larger population of non-T and non-B cells (CD3-B220-) associated with the splenomegaly and lymphadenopathy (Supplemental Figure $5 \mathrm{~B})$. The absolute numbers of $\mathrm{CD}^{+}$and $\mathrm{CD}^{+} \mathrm{T}$ cells were greater in $I t c h^{f l / f l} F o x p 3^{C r e}$ mice (Figure 2A, lower panel). To assess $\mathrm{T}$ cell homeostasis in $I t c f^{f / f l}$ Foxp $3^{\text {Cre }}$ mice, we examined the expression of $\mathrm{T}$ cell surface markers including CD44, CD62L, and CD69 in $\mathrm{YFP}^{-} \mathrm{CD} 4^{+} \mathrm{T}$ cells isolated from the spleens of $\mathrm{Itch}^{+/+}$Foxp $3^{\mathrm{Cre}}$ and Itch ${ }^{l / f l} \mathrm{Foxp} 3^{\mathrm{Cre}}$ mice. A higher percentage of conventional $\mathrm{CD} 4^{+} \mathrm{T}$ cells in Itch $f^{f / f l} \mathrm{Foxp} 3^{\mathrm{Cre}}$ mice exhibited an activated CD44 ${ }^{\mathrm{hi}} \mathrm{CD} 62 \mathrm{~L}^{\text {lo }}$ or $\mathrm{CD}_{69}{ }^{+}$phenotype compared with $\mathrm{Itch}^{+/+} \mathrm{Foxp} 3 \mathrm{Cre}$ controls (Figure $2 \mathrm{~B}$ ). In agreement with the Th2-type phenotype, $\mathrm{CD}^{+} \mathrm{T}$ cells from $I t c h^{f / f l} \mathrm{Foxp} 3^{\mathrm{Cre}}$ mice showed increased production of the Th2 cytokines IL-4, IL-5, and IL-10, whereas the production of IL-2, the Th1 cytokine IFN- $\gamma$, and the Th17 cytokine IL-17 was largely unchanged (Figure 2, C and D). In addition, we observed a selective increase of Th 2 cytokine production by $\mathrm{CD}^{+} \mathrm{T}$ cells in the lungs and peripheral lymph nodes of Itch $h^{f / f l} \mathrm{Foxp} 3^{\mathrm{Cre}}$ mice (Supplemental Figure $6, A$ and B). Interestingly, the production of IL- 17 by $\mathrm{CD}^{+}$ $\mathrm{T}$ cells was significantly increased in the lungs of Itchl/flf Foxp $3^{\mathrm{Cre}}$ mice compared with $\mathrm{Itch}{ }^{+/+} \mathrm{Foxp} 3^{\mathrm{Cre}}$ mice (Supplemental Figure $6 \mathrm{~A})$, implying that hyperproduction of IL-17 could contribute to the exaggerated lung inflammation observed in $\mathrm{Itch}^{f / f l} \mathrm{Foxp} 3^{\mathrm{Cre}}$ mice. Anti-CD3-stimulated Itch ${ }^{f / f l} \mathrm{Foxp} 3^{\mathrm{Cre}}$ splenocytes produced substantially more IL-4, IL-5, IL-13, and IL-10 cytokines than the control splenocytes (Supplemental Figure 7). Because IL-4-secreting Th2 cells are involved in class switching to IgG1 and IgE, we measured serum Ig isotypes by an ELISA assay. Consistent with the uncontrolled Th2 response, Itch ${ }^{f / f l} \mathrm{Foxp} 3^{\mathrm{Cre}}$ mice were found to produce highly increased levels of IgG1 and IgE compared with $\mathrm{Itch}^{+/+} \mathrm{Foxp} 3^{\mathrm{Cre}}$, whereas the serum IgA concentration was only modestly increased (Figure 2E). However, the amounts of the other Ig isotypes, including IgG2a, IgG2b, IgG3, and IgM were similar between $I t c b^{+/+} F o x p 3^{C r e}$ and $I t c h^{f / f l} F o x p 3^{C r e}$ mice (Figure 2E). Collectively, these results indicate that Treg cell-specific Itch deficiency leads to dysregulated Th2 responses.

Itch is dispensable for the suppressive activity and stability of Treg cells. To identify the underlying mechanisms responsible for the inflammatory disorder observed in $I t c h^{f l / f l} F o x p 3^{C r e}$ mice, we first sought to examine whether Treg cell homeostasis was impaired in the thymus and peripheral lymphoid organs of $I t c h^{f l / f l} F o x p 3^{C r e}$ mice. There was no significant difference in the proportion of Foxp3 ${ }^{+}$ thymocytes between $\mathrm{Itch}^{+/+} \mathrm{Foxp} 3^{\mathrm{Cre}}$ and $\mathrm{Itch} \mathrm{flf}^{\mathrm{fl} l \mathrm{Foxp}} 3^{\mathrm{Cre}}$ mice (Figure $3 \mathrm{~A})$. The absolute number of Treg cells was slightly higher in Itch $f^{f / f l}$ Foxp3 $3^{\mathrm{Cre}}$ mice than in $\mathrm{Itch}{ }^{+/+} \mathrm{Foxp} 3^{\mathrm{Cre}}$ mice (Figure 3C), but Itch deficiency did not change Foxp3 protein expression on a per-cell basis (Figure 3, A and B). These results imply that the inflammatory disorder in $I t c b^{f / f l} F o x p 3^{C r e}$ mice was not due to reduced numbers of Treg cells. We next examined whether Itch deficiency results in a general impairment of Treg suppressive function. An in vitro suppression assay showed that wild-type and Itch-deficient Treg cells had a similar ability to suppress the proliferation of naive $\mathrm{CD}^{+}$ $\mathrm{T}$ cells (Figure 4A). Furthermore, the addition of anti-IL-10 antibody to cultures resulted in an increase in cell division of naive $\mathrm{CD}^{+} \mathrm{T}$ cells at a ratio of 1 Treg cell to 25 naive $\mathrm{CD}^{+} \mathrm{T}$ cells (Supplemental Figure 8), implying that a similar mode of suppression is being used among wild-type and Itch-deficient Treg cells in vitro. To assess the in vivo suppressive function of Treg cells, we 
A

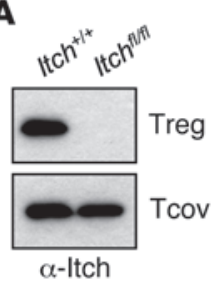

B

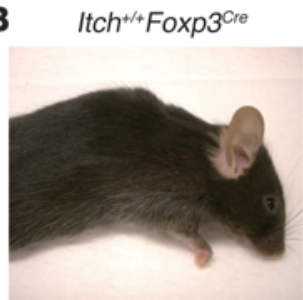

Itch $^{\text {HWI}}$ Foxp3 $3^{\text {cre }}$

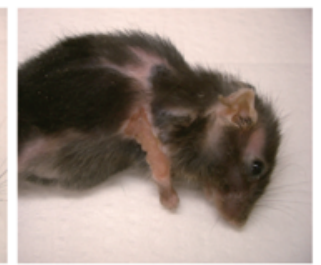

C

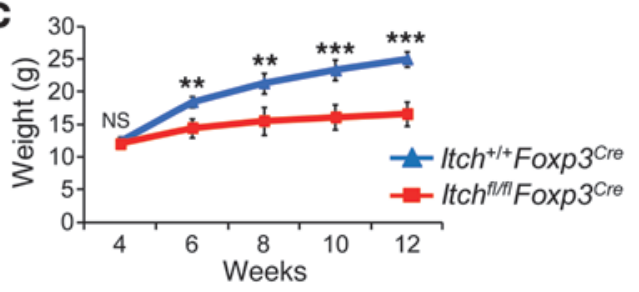

D

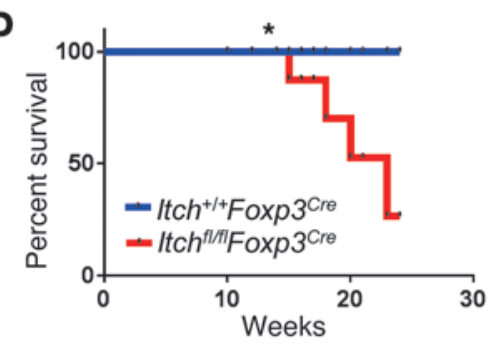

E

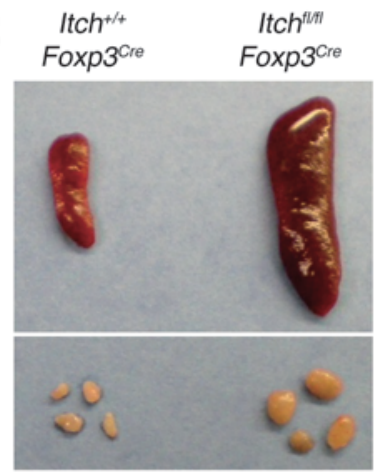

F

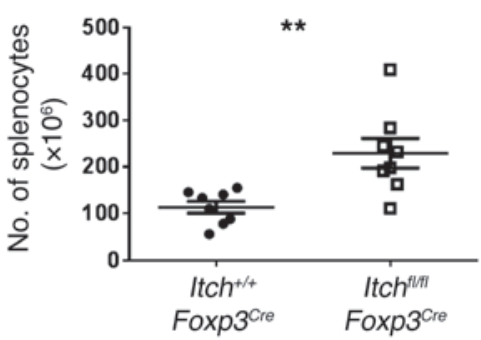

G
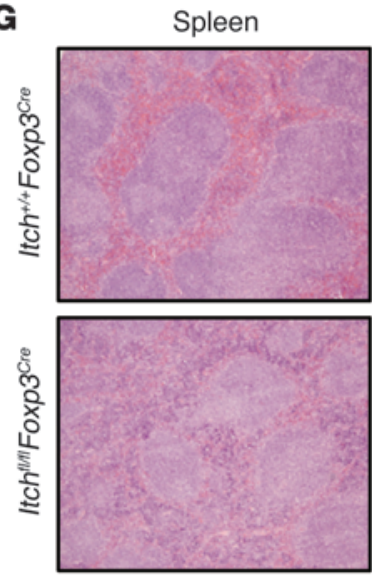

H
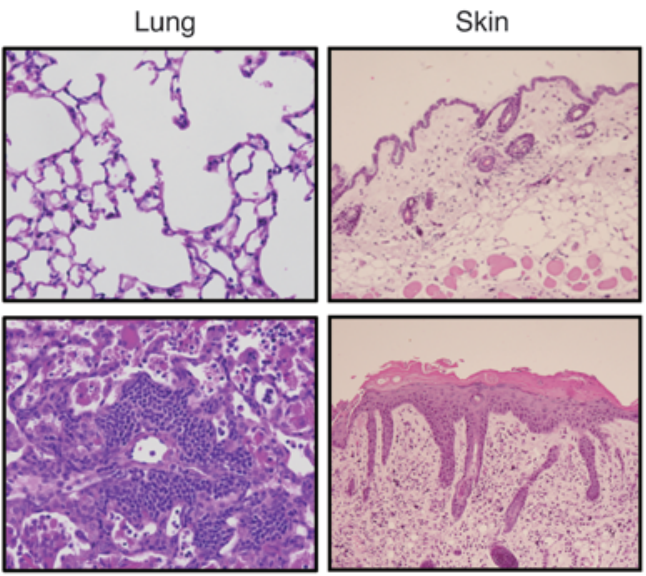
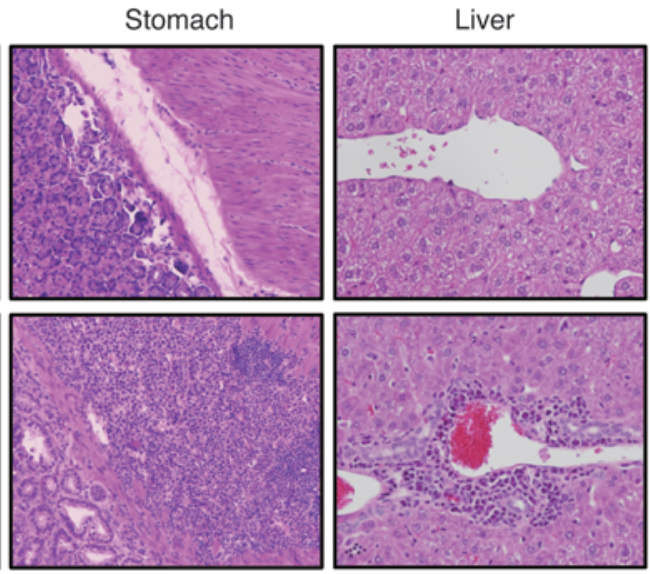
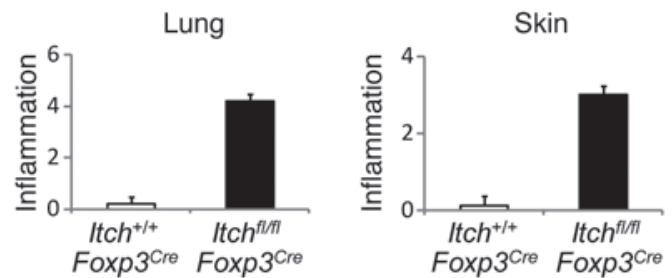

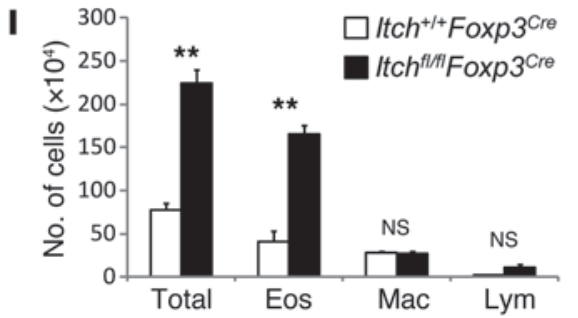

$\mathbf{J}$

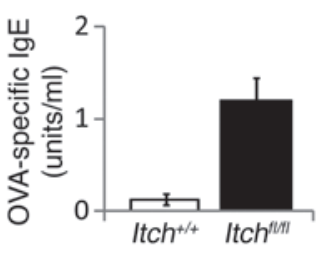

\section{Figure 1}

Treg cell-specific Itch deletion causes severe inflammation. (A) Immunoblot analysis of Itch in splenic CD4+YFP- conventional T cells (Tcov) and

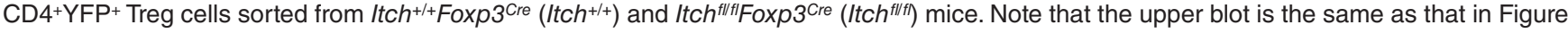

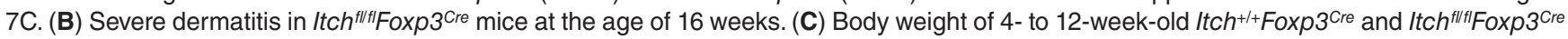
mice. (D) Kaplan-Meier survival curve of Itch ${ }^{+/+}$Foxp3 $3^{C r e}$ and $I t c h^{f / f t} F o x p 3^{C r e}$ mice. (E) Splenomegaly and lymphadenopathy in Itch ${ }^{f / / f I} F o x p 3^{C r e}$ mice. (F) Spleen cellularity in Itch ${ }^{+/+}$Foxp3 ${ }^{C r e}$ mice (black circles) and Itch ${ }^{\text {fllfIFoxp3 }}$ Cre mice (white squares). (G) Representative H\&E-stained tissue sections from 10-week-old Itch ${ }^{+/+}$Foxp3 $3^{\text {Cre }}$ and Itch $h^{f / f}$ Foxp3 $3^{\text {Cre }}$ mice. Original magnification, $\times 200$ and $\times 100$ (skin). (H) Inflammation scores.

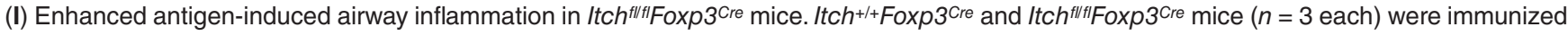
i.p. with OVA in alum. Eighteen days later, mice were challenged by i.n. administration of OVA for 4 consecutive days. Total numbers of eosinophils (Eos), monocytes/macrophages (Mac), and lymphocytes (Lym) were calculated in BAL fluid 24 hours after the last OVA challenge. (J) Serum levels of OVA-specific IgE were measured by an ELISA assay. Data are compiled from two independent experiments with four mice per group. Error bars indicate the mean $( \pm \mathrm{SD}) .{ }^{\star} P<0.05 ;{ }^{* \star} P<0.01$; ${ }^{* \star} P<0.001$ (unpaired 2-tailed Student's $t$ test). 

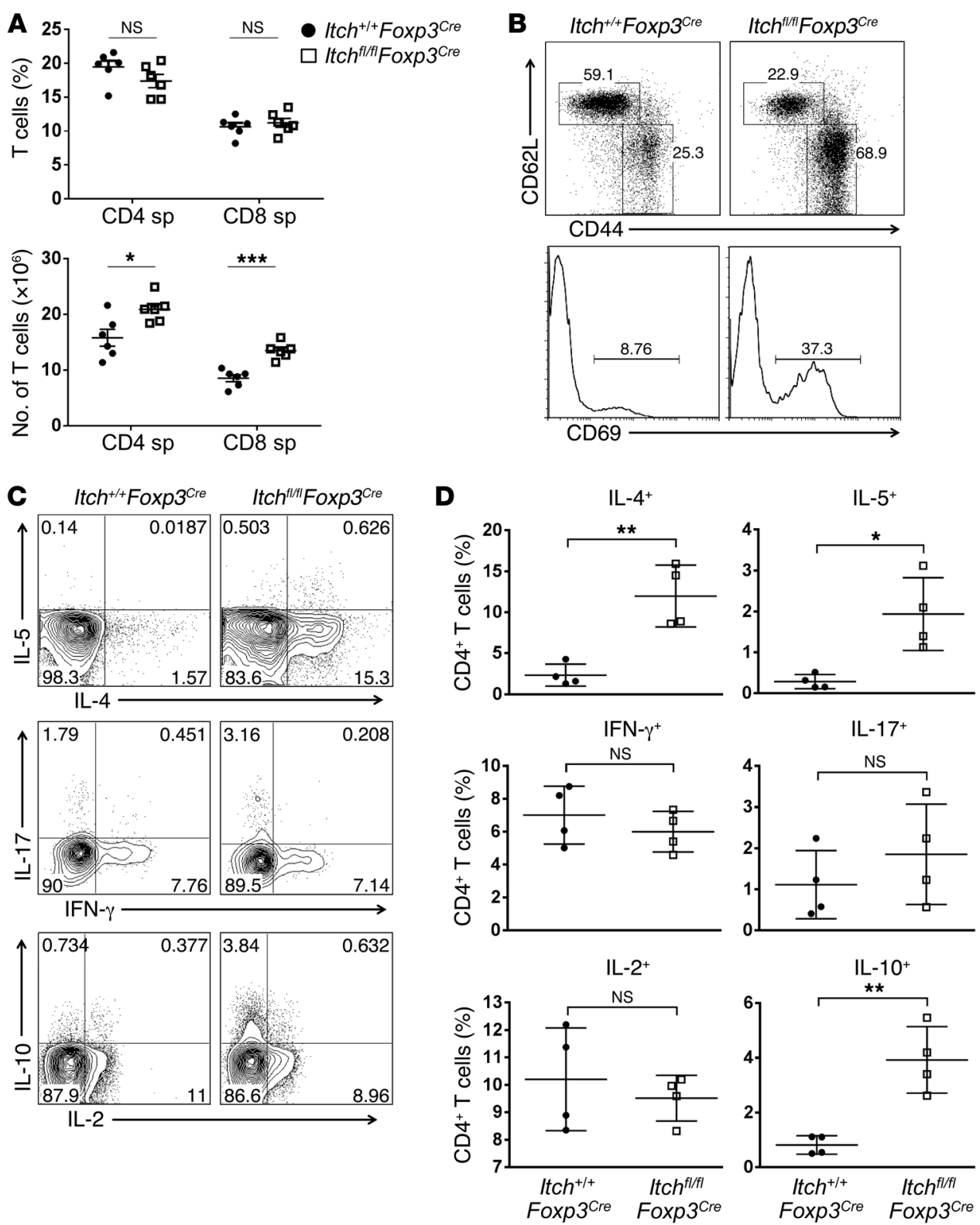

\section{Figure 2}

Selective dysregulation of Th2 responses in Itch ${ }^{\text {fllt }}$ Foxp3 ${ }^{\text {Cre }}$ mice. (A) The percentage and number of $\mathrm{CD} 4^{+}$ and $C D 8^{+} \mathrm{T}$ cells in the spleen (sp) of Itch $h^{\text {fl/II }}$ Foxp $3^{\text {Cre }}$ (white squares) and Itch ${ }^{+/+}$Foxp3 ${ }^{\text {Cre }}$ (black circles) littermates. (B) Flow cytometric analysis of CD44, CD62L, and CD69 expression on conventional $\mathrm{CD}^{+} \mathrm{T}$ cells in 8-week-old Itch ${ }^{\text {fl/fl}}$ Foxp3Cre mice and Itch ${ }^{+/+}$Foxp3 ${ }^{\text {Cre }}$ littermates. One representative experiment of three independent experiments is shown. (C) Flow cytometric analysis of cytokine production by splenic CD4 ${ }^{+} \mathrm{T}$ cells from 8-week-old Itch ${ }^{f / f t}$ Foxp3 ${ }^{\text {Cre }}$ mice and $/ t_{c h}{ }^{+/+}$Foxp3 3 re littermates. Splenocytes were stimulated with PMA $(100 \mathrm{ng} / \mathrm{ml})$ and ionomycin $(1 \mu \mathrm{g} / \mathrm{ml})$ in the presence of Golgi stop for 4 hours. (D) Frequency of CD4+ $\mathrm{T}$ cells producing IL-4, IL-5, IFN- $\gamma$, IL-17, IL-2, or IL-10. Data represent the mean \pm SEM. (E) Serum samples were obtained from 8- to 12-week-old Itch $h^{\text {fllfl }}$ Foxp3 ${ }^{\text {Cre }}$ mice and tch $^{+/+}$Foxp3 $3^{\text {Cre }}$ littermates $(n=6)$. Itch $h^{f / / f l}$ Foxp3 ${ }^{\text {Cre }}$ (white squares) and Itch ${ }^{+/+}$Foxp3 $3^{\text {Cre }}$ mice (black circles). Total amounts of each Ig subclass were determined by ELISA using isotype-specific antibodies. ${ }^{*} P<0.05 ;{ }^{* *} P<0.01 ;{ }^{* *} P<0.001$ (unpaired 2-tailed Student's $t$ test).

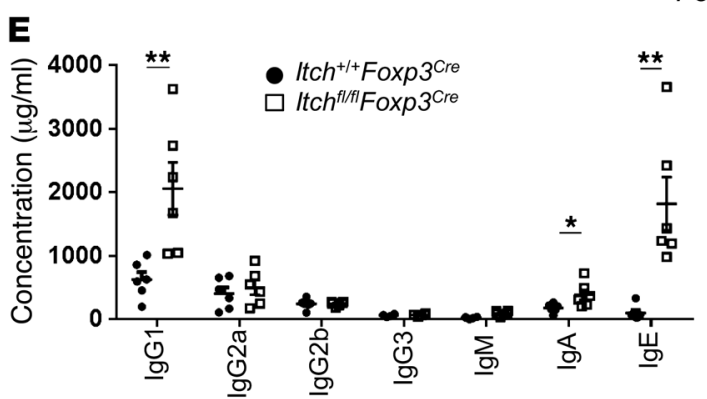

used an adoptive transfer model of colitis induced by transferring naive $\mathrm{CD} 4^{+}$cells into recombination-activating gene 1 -deficient $\left(\right.$ Rag $\left.1^{-/}\right)$mice. The transfer of wild-type naive $\left(\mathrm{CD} 45.1^{+} \mathrm{CD} 25^{-}\right.$ $\mathrm{CD}_{5}$ RB ${ }^{\mathrm{hi}}$ ) $\mathrm{CD}^{+} \mathrm{T}$ cells alone elicited colitis in $\mathrm{Rag}^{-/-}$recipient mice, manifested by gradual weight loss (Figure 4B), splenomegaly, and lymphadenopathy (Figure 4C). However, the cotransfer of either CD45.2+ wild-type or Itch-deficient Treg cells, together with the naive $\mathrm{CD} 45 \mathrm{RB}^{\text {hi }} \mathrm{CD}^{+} \mathrm{T}$ cells, effectively suppressed the autoimmune phenotype of naive $\mathrm{CD}^{+} \mathrm{T}$ cells. Recipient mice given either wild-type or Itch-deficient Treg cells remained healthy without losing body weight. Histological analysis of recipients of wild-type or Itch-deficient Treg cells also showed a low degree of inflammation compared with that of mice that did not receive Treg cells (Figure 4D), suggesting that Itch-deficient Treg cells 

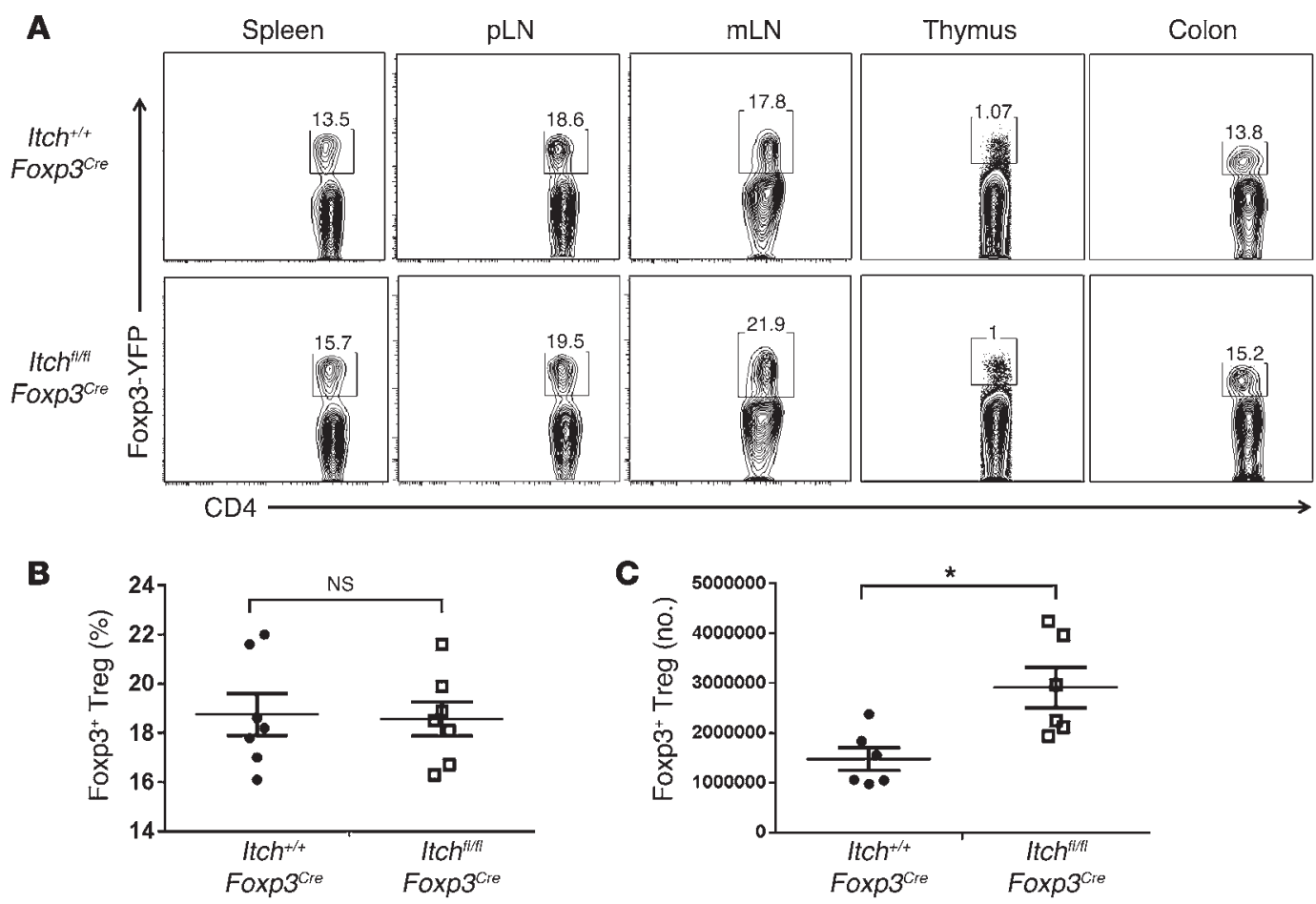

Figure 3

Itch is dispensable for Treg cell homeostasis. (A) Percentages of YFP+ Treg cells among CD4+ T cells from spleen, peripheral lymph nodes (pLN), mesenteric lymph nodes $(\mathrm{mLN})$, thymus, and colon of $I t c h^{f l / f} F o x p 3^{C r e}$ and $I t c h^{+/+} F o x p 3^{C r e}$ mice. (B) Frequency of YFP ${ }^{+}$cells among $\mathrm{CD}^{+}{ }^{+}$cells in

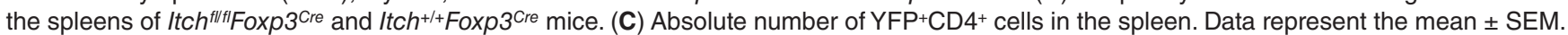
${ }^{\star} P<0.05$ (unpaired 2-tailed Student's $t$ test).

have competent immune-suppressive activity in vivo. In addition, we analyzed the percentage and total number of the CD45.1 $1^{+}$progeny of $\mathrm{CD} 4^{+} \mathrm{CD} 45 \mathrm{RB}{ }^{\text {hi }} \mathrm{T}$ cells and the $\mathrm{CD} 45.2^{+}$progeny of Treg cells in the $\mathrm{Rag}^{-/-}$recipients. In the presence of either wild-type or Itch-deficient Treg cells, naive $\mathrm{T}$ cell expansion was significantly reduced (Figure 4E). The ratios of CD $45.1^{+}$cells/CD45.2 $2^{+}$cells in the recipient mice given Itch-deficient Treg cells were moderately decreased compared with those in the mice that received wildtype Treg cells in all organs analyzed (Supplemental Figure 9A). This phenomenon was likely due to the enhanced expansion of Itch-deficient Treg cells in the Rag $1^{-/-}$hosts, since the total number of Itch-deficient Treg cells was increased compared with that of wild-type Treg cells (Figure 4E). We next examined whether the increased expansion of Itch-deficient Treg cells in lymphopenic hosts was associated with their Foxp3 instability. The wild-type and Itch-deficient Treg cells obtained from the Rag1/-- hosts displayed similar amounts of Foxp3 (Figure 4F), suggesting that Itch was dispensable for maintaining Foxp3 stability.

To further reinforce this conclusion, we examined the stability of Treg cells using a genetic fate-mapping approach: Itch ${ }^{f l / f l} F o x p 33^{C r e}$ mice were crossed with Rosa26 red fluorescent protein (RFP) Cre reporter mice (R26R), in which RFP is expressed only after Cremediated excision of a loxP-flanked stop codon (31). The loss of YFP expression in the $\mathrm{RFP}^{+} \mathrm{CD} 4^{+} \mathrm{T}$ cells marks the generation of latent or ex-Treg cells, which was reported in autoimmune mice (18). By using this fate-mapping approach, we examined whether Itch deficiency accelerates the generation of YFP-RFP ${ }^{+}$ex-Treg cells under inflammatory conditions. We observed that Itch $f^{f / f l}$ Foxp $3^{C r e}$ 4-month-old mice with a severe inflammatory phenotype had a similar percentage of ex-Treg cells in their peripheral lymphoid organs compared with control $\mathrm{Itch}^{+/+} \mathrm{Foxp}^{\mathrm{Cre}}$ mice (Figure 4G). Similar results were obtained when we expanded the sorted $\mathrm{YFP}^{+} \mathrm{RFP}^{+}$ Treg cells in vitro using anti-CD3/CD28 antibodies and IL-2. After 5 days of culture, the percentage of $\mathrm{YFP}^{-} \mathrm{RFP}^{+}$ex-Treg cells was comparable between wild-type and Itch-deficient Treg cells (Supplemental Figure 9B). Furthermore, when we cotransferred violet dye-labeled wild-type $\mathrm{YFP}^{+} \mathrm{RFP}^{+}$Treg and efluor670 dye-labeled Itch-deficient $\mathrm{YFP}^{+} \mathrm{RFP}^{+}$Treg cells into Rag $1^{-/-}$recipient mice, we found that a fraction of the Treg cells lost Foxp3 expression (YFP$\mathrm{RFP}^{+}$population), and this occurred to a similar degree in wild-type Treg and Itch-deficient Treg cells (Supplemental Figure 10). Collectively, these results indicate that Itch deficiency does not affect the suppressive function and stability of Treg cells.

Acquisition of effector function by Itch-deficient Treg cells. We next examined whether Itch deficiency in Treg cells resulted in unrestrained cytokine production in Treg cells. Strikingly, Bio-Plex cytokine array analysis showed that anti-CD3/CD28-stimulated Itch-deficient Treg cells produced large amounts of Th2 cytokines, including IL-4, IL-5, IL-10, and IL-13 (Figure 5A and Supplemental Figure 11). The production of IL- 4 by Itch-deficient Treg cells was confirmed by intracellular cytokine staining and RT-PCR analysis (Supplemental Figure 12, A and B). As expected, control wild-type Treg cells did not produce noticeable amounts of proinflammatory cytokines. Furthermore, we found that the increase in Th2 cytokine production was excessively augmented in $\mathrm{Itch}^{-/}$ Foxp3-negative ex-Treg cells, as measured by Bio-Plex cytokine 
A
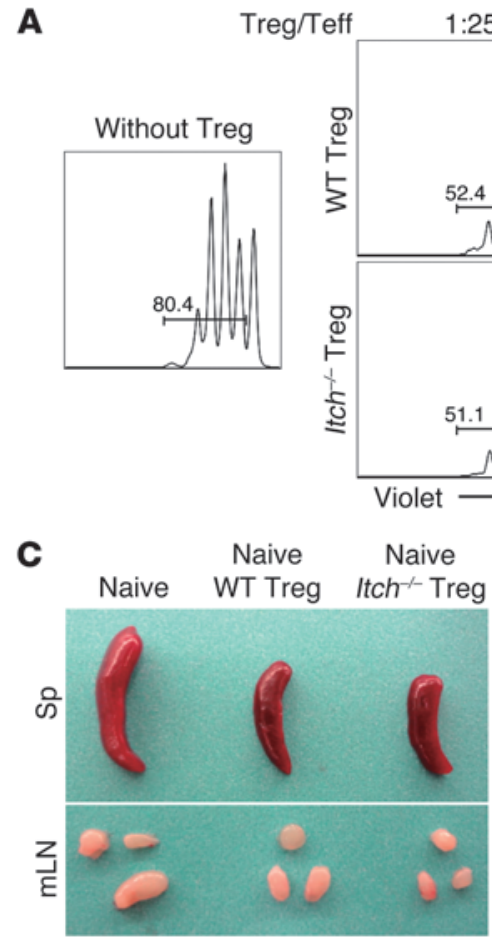

$\mathbf{E}$

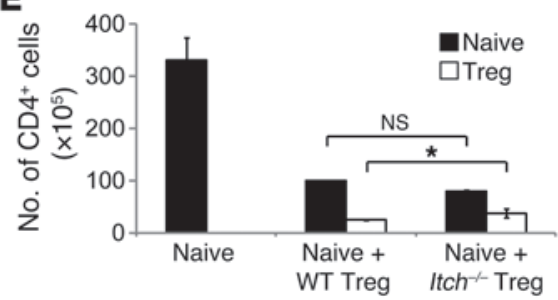

D

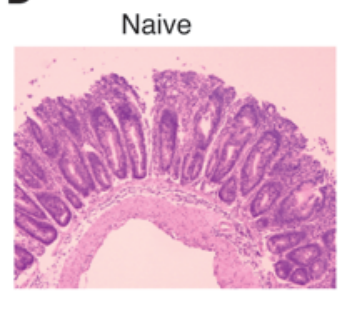

$1: 1$

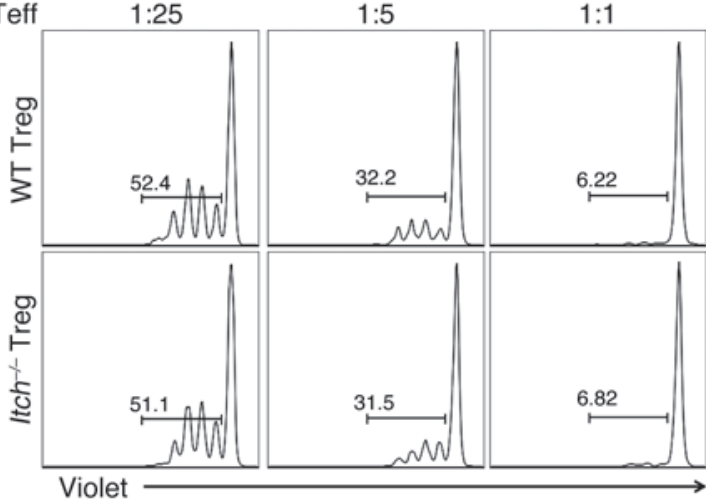

B

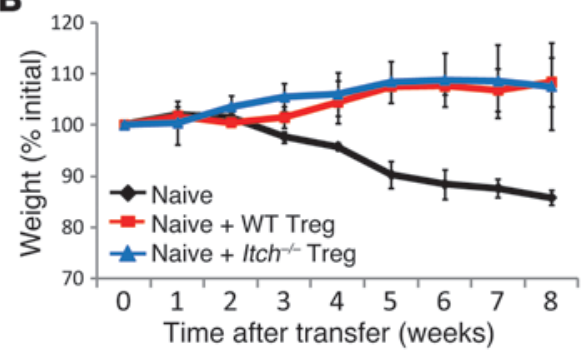

G
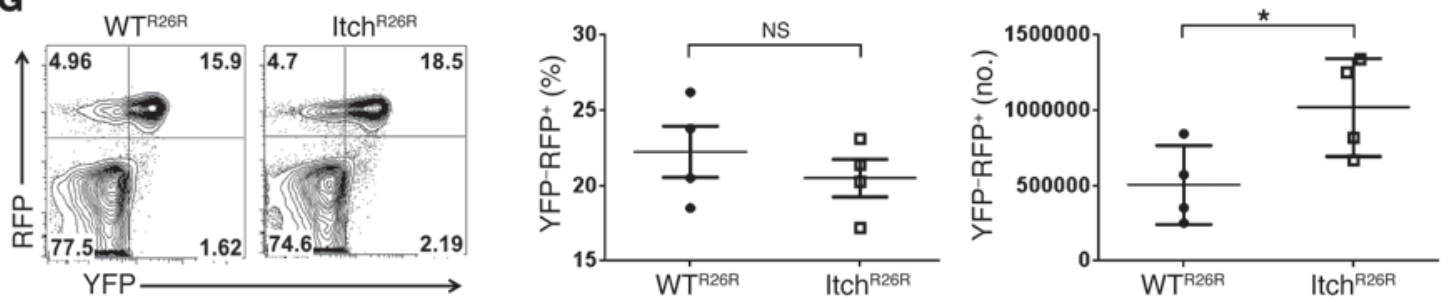

Figure 4

Itch-deficient Treg cells have intact in vivo and in vitro suppressive activity. (A) CD25-CD4+ $T$ cells were sorted from wild-type C57BL/6 mice and labeled with violet dye. Labeled CD4 ${ }^{+} T$ cells were then activated by anti-CD3 antibodies plus irradiated APCs in the presence of various ratios of Treg cells sorted from Itch fl/filFoxp3 $3^{\text {Cre }}$ and Itch ${ }^{+/+}$Foxp3 ${ }^{C r e}$ mice. Teff, effector T cells. (B-F) Rag 1-/- mice were given sorted wild-type or Itchdeficient $\left(C D 45.2^{+}\right)$Treg cells, together with CD4+CD45RB hi (CD45.1 $1^{+}$) naive T cells, or CD4+CD45RB hi (CD45. $\left.1^{+}\right)$naive T cells alone. (B) Weight loss of individual mice was monitored every week for 2 months. Data are compiled from three independent experiments with four mice each. Error bars indicate the mean $( \pm \mathrm{SD})$. (C) Photograph of spleen $(\mathrm{Sp})$ and mesenteric lymph nodes $(\mathrm{mLN})$. (D) H\&E staining of colon sections of recipient mice at 8 weeks after adoptive transfer. (E) Absolute number of $C D 4+C D 45.1^{+}$and $C D 4+C D 45.2^{+} T$ cells in the spleens of the recipient mice at 8 weeks after adoptive transfer. Error bars indicate the mean $( \pm S D)$. (F) Foxp3 expression by CD4+CD45.2+ cells from Rag1-/- recipient mice. Data are representative of three independent experiments. (G) Flow cytometric analysis of YFP and RFP expression in CD4+ ${ }^{+}$cells of 4-monthold WTR26R and Itch ${ }^{\text {R26R }}$ mice (left panel). Percentages of ex-Treg cells (YFP-RFP+) in total RFP+ cells (YFP+RFP+ and YFP-RFP+) are indicated (middle panel). Absolute number of ex-Treg cells (YFP-RFP+) cells in the spleen $(n=4)$ (right panel). ${ }^{*} P<0.05$ (unpaired 2-tailed Student's $t$ test).

array analysis (Figure 5B) and flow cytometry (Supplemental Figure 12C). Flow cytometric analysis showed that Itch-deficient Treg cells have an activated phenotype with reduced CD62L and increased CD44 expression (Figure 5C). Compared with wild-type
Treg cells, Itch-deficient Treg cells expressed high levels of GITR (TNFRSF18) and ICOS (Figure 5D). The levels of CD25 (IL2RA) and CTLA4 were not altered in Itch-deficient Treg cells (Figure 5D), consistent with a potent immunosuppressive activity of 


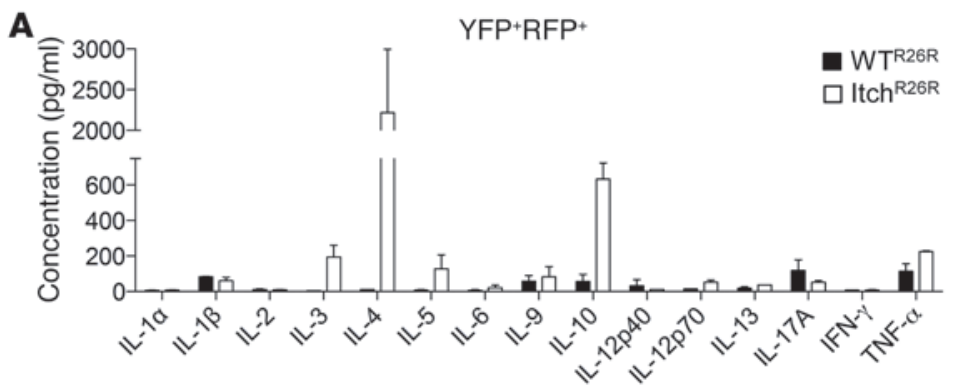

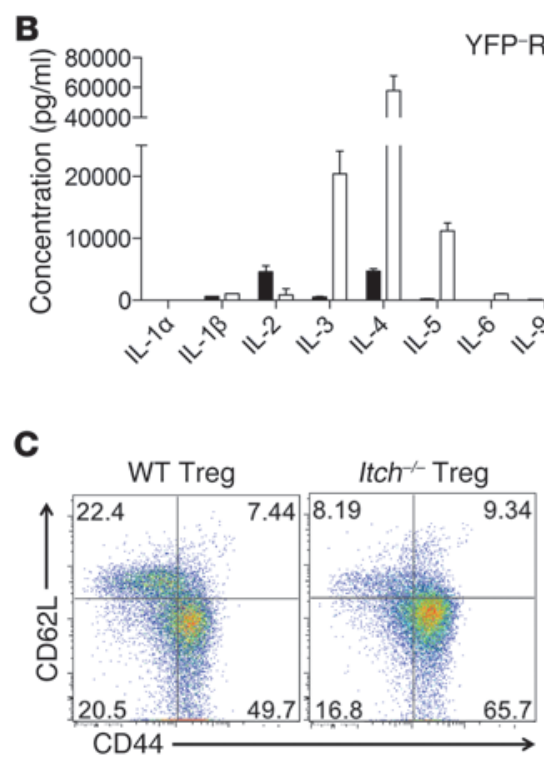

$\mathbf{E}$

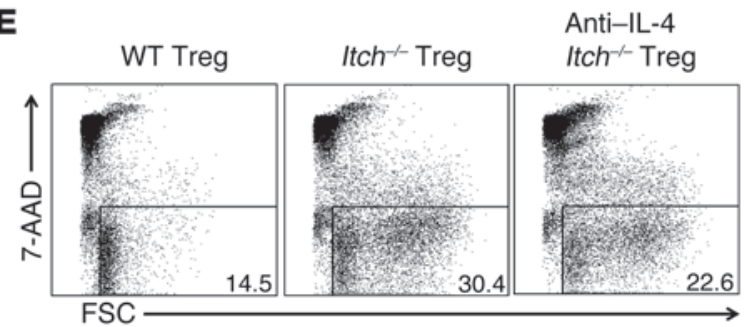

D

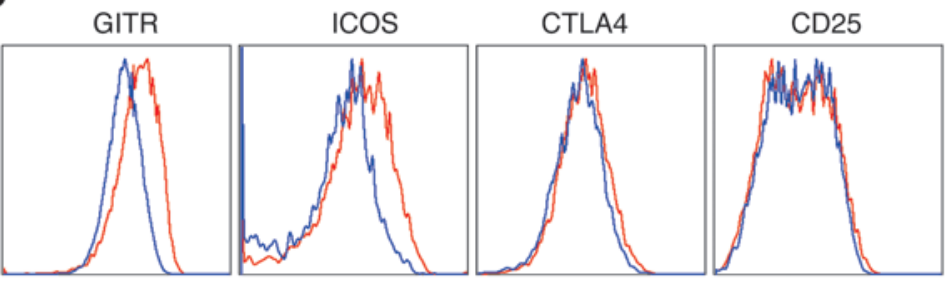

$\mathbf{F}$

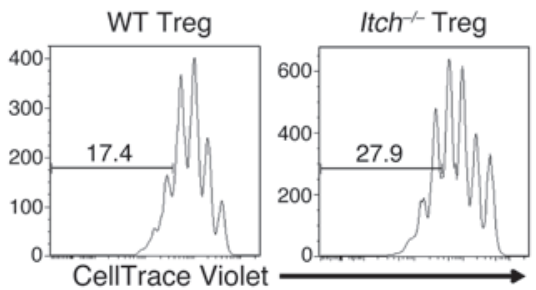

Figure 5

Loss of Itch in Treg cells resulted in the acquisition of Th2-like properties. (A and B) FACS-sorted CD4+YFP+RFP+ Treg cells (A) or CD4+ ${ }^{+}$YFP$\mathrm{RFP}^{+}$ex-Treg cells (B) from the spleens of WTR26R and Itch ${ }^{\mathrm{R} 26 \mathrm{R}}$ mice were stimulated with anti-CD3 and anti-CD28 antibodies for 48 hours in vitro. Cytokine production was measured by Bio-Plex multicytokine assay. Data are compiled from three independent experiments. Error bars indicate the mean ( \pm SD). (C) Flow cytometric analysis of CD62L and CD44 expression on YFP+CD4+ Treg cells in 8-week-old Itch ${ }^{\text {fllfl} F o x p 3 C r e ~ m i c e ~ a n d ~}$ Itch ${ }^{+/+}$Foxp3 3 re littermates. (D) Expression of Treg cell-associated molecules (GITR, ICOS, CTLA4, and CD25) on wild-type and Itch-deficient Treg cells. (E) Flow cytometric analysis of FACS-sorted CD4+YFP+ Treg cells stimulated with anti-CD3 and anti-CD28 antibodies for 2 days in the absence of IL-2 (upper panel). Anti-IL-4 antibody was added as indicated at $10 \mu \mathrm{g} / \mathrm{ml}$. Percentages of events in the live gates (7-AAD ${ }^{10}$ and FSChi) by flow cytometric analyses are shown in the bar graph (lower panel). (F) Violet dye-labeled FACS-sorted YFP+ Treg cells were stimulated with anti-CD3/CD28 antibodies for 4 days in the presence of IL-2. Cell division was analyzed by flow cytometry. All results are representative of at least three experiments. ${ }^{*} P<0.05$ (unpaired 2-tailed Student's $t$ test).

Itch-deficient Treg cells. To determine whether loss of Itch in Treg cells plays a role in their survival, we next analyzed the population of dead cells by 7-AAD staining and FSC hi gating after culturing sorted Treg cells for 2 days, without IL-2. Itch-deficient Treg cells displayed increased resistance to activation-induced cell death (Figure 5E). The addition of anti-IL-4 antibodies to the culture medium did not significantly reduce the Treg cells' resistance to cell death, suggesting that the enhanced survival of Itch-/- Treg cells is not strictly dependent on IL-4. In addition to enhancing their survival, Itch deficiency affected the proliferation of Treg cells. Analysis of individual cell division of violet dye-labeled Treg cells showed that Itch-deficient Treg cells divided much more rapidly compared with wild-type Treg cells in the absence or presence of IL-2 (Figure 5F and data not shown). We next examined whether Itch deficiency in Treg cells results in dysregulated Th2 responses in vivo using an OVA-induced airway inflammation model. Wild- 
A

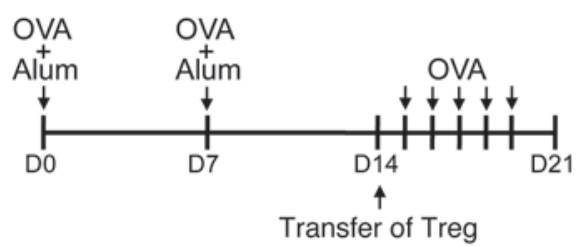

B

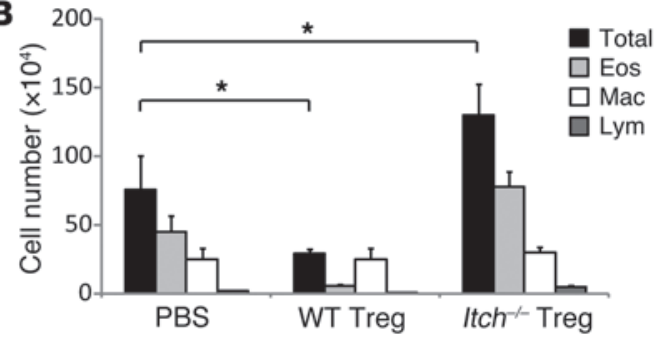

C

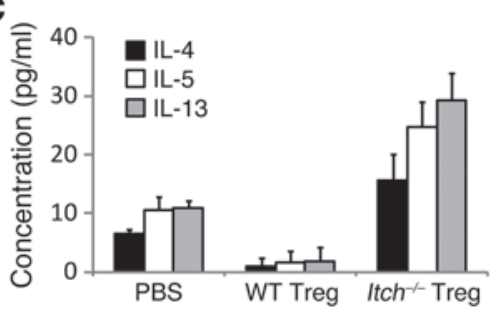

D

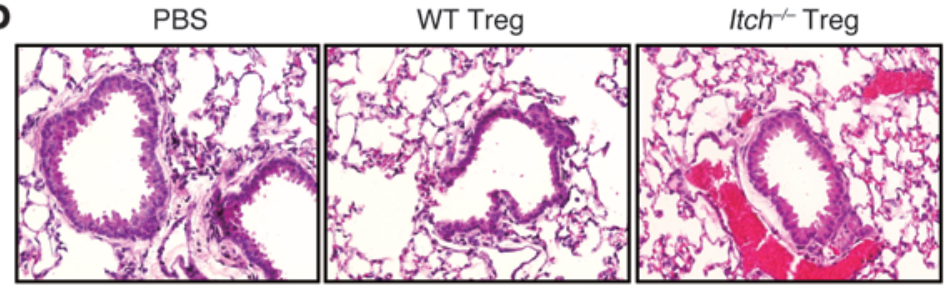

Figure 6

Itch-deficient Treg cells promoted higher Th2 responses in an OVA-induced airway inflammation model. (A) Schematic immunization protocol.

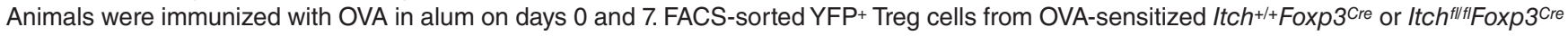
mice were injected into the sensitized wild-type B6 mice on day 14. PBS was injected as a control. One day after Treg transfer, recipient mice were challenged i.n. with OVA protein for 5 consecutive days. (B) Total numbers of eosinophils, monocytes/macrophages, and lymphocytes were calculated in BAL fluid 48 hours after the last OVA challenge. (C) IL-4, IL-5, and IL-13 concentrations in BAL fluid were measured by ELISA. (D) Lung tissue sections were stained with H\&E. Data are compiled from two independent experiments with two mice per group. Original magnification, $\times 200$. Error bars indicate the mean $( \pm \mathrm{SD}) .{ }^{*} P<0.05$ (unpaired 2-tailed Student's $t$ test).

type recipient mice, as well as $I t c b^{+/+}$Foxp $3^{\text {Cre }}$ and Itchflflf Foxp $3^{\text {Cre }}$ mice, were primed with OVA in alum on days 0 and 7 . Prior to OVA challenge, Treg cells isolated from OVA-immunized $\mathrm{Itch}^{+/+}$ Foxp $3^{\mathrm{Cre}}$ and Itch ${ }^{f / f l} \mathrm{Foxp} 3^{\mathrm{Cre}}$ mice were injected i.p. into OVA-immunized wild-type recipient mice (Figure 6A). We observed decreased inflammation and decreased numbers of allergic inflammatory cells in mice transferred with wild-type Treg cells compared with PBS-treated mice (Figure 6, B-D), indicating that wild-type Treg cells functioned to suppress allergic airway inflammation. In contrast, lung inflammation was increased in the recipient mice transferred with Itch-deficient Treg cells compared with that observed in PBS-treated mice, as evidenced by increased airway eosinophilia and elevated amounts of Th2 cytokines in the BAL fluid (Figure $6, \mathrm{~B}-\mathrm{D})$. These data indicate that Itch-deficient Treg cells exacerbate Th2 immune responses in allergic inflammation in vivo. Altogether, these findings indicate that Itch plays an important role in preventing the acquisition of Th2-like properties by Treg cells.

GATA3 and STAT6 control pathogenic Th2 responses in Itch-deficient Treg cells. To further investigate the molecular mechanism by which Itch regulates the function of Treg cells, we examined the effect of Treg-specific Itch deficiency on the expression of transcription factors that drive the Th2 differentiation program. Consistent with the hyperproduction of Th2 cell cytokines, mRNA expression of Th2 transcription factor Gata3 and c-Maf was significantly increased in Itch-deficient Treg cells, whereas the level of Th1 transcription factor Tbx21 was unchanged. Itch-deficient Treg cells showed a lower expression of the Th17 transcription factor Rorc compared with wild-type Treg cells (Figure 7A). Increased GATA3 expression in Itch-deficient Treg cells was also seen by flow cytometry (Figure 7B). In addition, immunoblot analysis showed that GATA3 expression was markedly increased in TCR-stimulated Itch-deficient Treg cells compared with wild-type Treg cells (Figure
7C). TCR stimulation also induced Tyr-641 phosphorylation of the signal transducer and activator of transcription 6 (STAT6) in Itch-deficient Treg cells (Figure 7, B and C). To investigate whether the inhibition of Th2 transcription factors is able to rescue the Th2 phenotype in Itch-deficient Treg cells, we generated retroviral vectors expressing both mAmetrine and an shRNA targeting Gata3 or Stat6. Knockdown efficiency was confirmed by RT-PCR (data not shown). Bone marrow cells isolated from $\mathrm{Itch}^{\mathrm{fl} / f l} \mathrm{Foxp} 3^{\mathrm{Cre}}$ mice were transduced with the retrovirus carrying each of the shRNAs or the control shRNA and were then transplanted into lethally irradiated C57BL/6 mice. Eight weeks later, newly reconstituted mAmetrine ${ }^{+} \mathrm{YPP}^{+} \mathrm{CD} 4^{+}$Treg cells were sorted from the spleens and lymph nodes of bone marrow chimeric mice and then stimulated with anti-CD3/CD28 antibodies in vitro. The elevated production of Th2 cytokines in Itch-deficient Treg cells was completely inhibited by Gata3 or Stat6 knockdown, as measured by an ELISA assay (Figure 7D), indicating that unrestrained STAT6 activation and GATA3 expression in Itch-deficient Treg cells are associated with dysregulated Th2 responses.

Itch-deficient Treg cells instruct Th2 cell differentiation in vitro. Since Treg cells constitute a small population, we reasoned that Th2 cytokine production by Itch-deficient Treg cells does not fully account for the pathogenic Th 2 responses observed in $I t c b^{f l f l}$ Foxp $3^{\text {Cre }}$ mice. We thus hypothesized that the cytokines secreted from Treg cells instruct naive $\mathrm{T}$ cells to develop into Th2 cells, thereby promoting the development of pathogenic Th2 responses and associated disease. To test this, we stimulated naive $\mathrm{CD} 4^{+} \mathrm{T}$ cells in the presence of culture supernatants from Itch-deficient Treg cells and found that they instructed naive $\mathrm{T}$ cells to differentiate into IL-4-producing effector cells (Figure 8A). The mRNA levels of Il4 and Il5, but not Ifng, were also increased in these cells (Figure 8B). In addition, RT-PCR analysis showed the upregulation of Gata3 concomitant with the sup- 
A
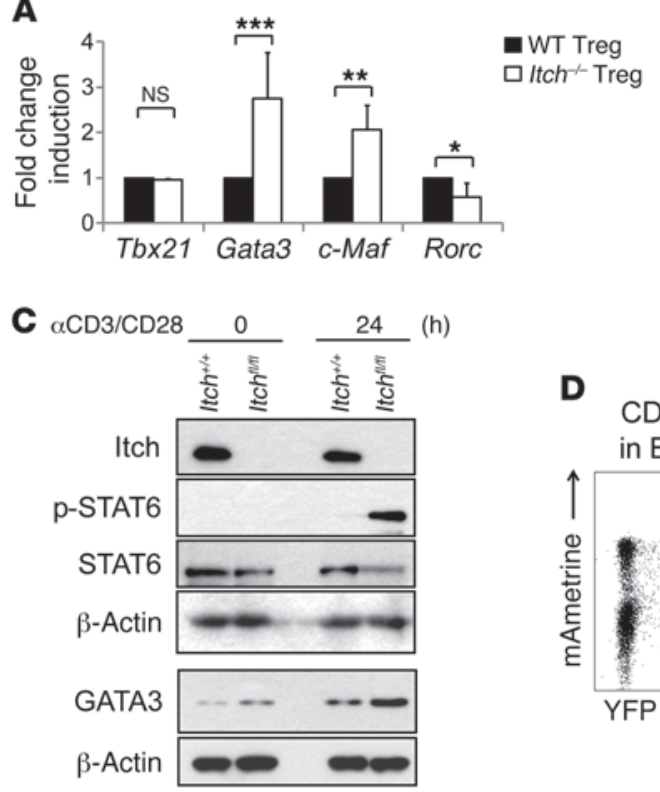

B

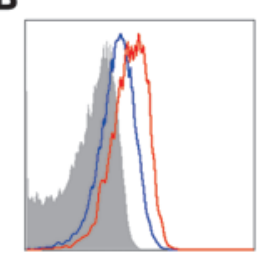

GATA3 $\alpha \mathrm{CD} 3 / \mathrm{CD} 28$

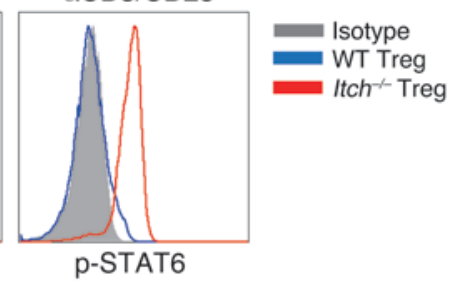

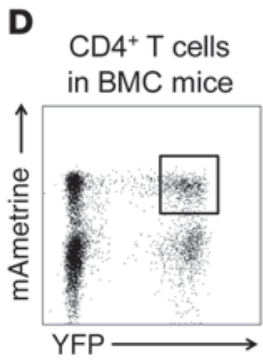

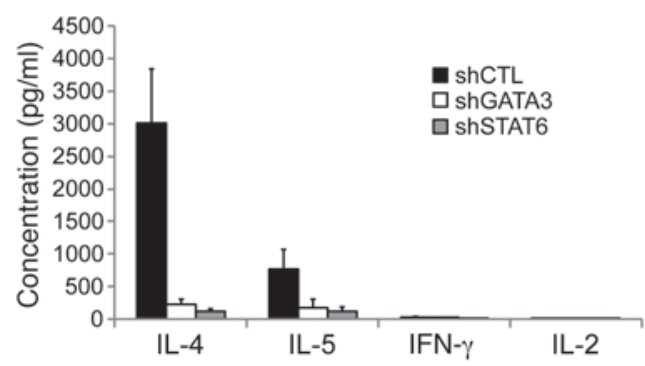

\section{Figure 7}

Inhibition of Th2 transcription factors rescued aberrant cytokine production of Itch-deficient Treg cells. (A) Relative mRNA expression amounts of Tbx21, Gata3, c-Maf and Rorc in sorted CD4+YFP+ Treg cells from Itch ${ }^{\mathrm{fl} / \mathrm{fl}} \mathrm{Foxp} 3^{\mathrm{Cre}}$ mice and Itch ${ }^{+/+}$Foxp $3^{\mathrm{Cre}}$ littermates. Results of three experiments are shown as the mean \pm SD values. (B) FACS-sorted CD4+ YFP+ Treg cells were fixed and stained with either anti-GATA3 antibody or an isotype-matched control Ig. For phospho-STAT6 detection (p-STAT6), FACS-sorted CD4+YFP+ Treg cells were stimulated with anti-CD3/ CD28 antibodies for 2 hours, followed by staining with antiphospho-STAT6 antibody. (C) Immunoblot analysis of wild-type and Itch-deficient Treg

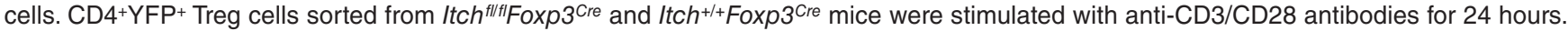
Cell lysates were separated by SDS-PAGE and immunoblotted with the indicated antibodies. The blot for Itch is the same as that in Figure $1 \mathrm{~A}$. (D) Flow cytometric analysis of YFP and mAmetrine expression in CD4+ $T$ cells in the bone marrow chimeric mice (BMC). shRNA-expressing bone marrow chimeric mice were generated by reconstituting retrovirally transduced bone marrow cells from Itch ${ }^{f / f I}$ Foxp $3^{\text {Cre }}$ mice into lethally irradiated recipient mice. YFP and mAmetrine double-positive CD4+ T cells were sorted and activated with anti-CD3/CD28 antibodies for 2 days in vitro. Cytokine concentration in the culture supernatants was measured by an ELISA assay. Data are compiled from two independent experiments with four mice per group. Error bars indicate the mean ( \pm SD). ${ }^{\star} P<0.05 ;{ }^{\star \star} P<0.01 ;{ }^{\star \star \star} P<0.001$.

pression of Tbx21 in the naive $T$ cells when cultured with the supernatants from Itch-deficient Treg cells (Figure 8C). The addition of anti-IL-4 antibody into the supernatants abolished Th2 instruction, indicating that IL-4 from Itch-deficient Treg cells plays a critical role in the instruction of Th2 cell differentiation.

\section{Discussion}

Previous studies including our own have demonstrated that Itch deficiency results in the reduction of Foxp3 expression and decreased induction and differentiation of iTreg cells $(28,29)$. It was striking to see that Foxp3-restricted deletion of Itch did not affect Foxp3 expression or the development and differentiation of nTreg cells. One explanation is that the differentiation of iTreg cells requires the stimulation of TGF- $\beta$ signaling (8), which may not be necessary for nTreg cells (32). The increased IL-4 production accounted for the resistance of naive $\mathrm{CD}^{+}$cell conversion into iTreg cells (29), which is consistent with the observation that IL-4-driven GATA3 expression counteracts Foxp3 expression in iTreg cells $(33,34)$. However, recent genetic studies clearly showed that GATA3 expression is required for nTreg development and function $(20,35)$. It would be reasonable to reconcile the different roles of Itch in iTreg and nTreg cells by the fact that the iTreg studies used $\mathrm{CD}^{+} \mathrm{T}$ cells from Itchy mutant mice, which are chronically activated and are therefore resistant to Foxp3 induction.
Thus, the present study potentially highlights the importance of studying the intrinsic molecular regulation of Foxp3 using committed nTreg cells instead of iTreg cells from inflamed mice, which may not reveal the true biological relevance of Foxp3 regulation.

Foxp3-restricted deletion of Itch leads to multiorgan inflammation and skin disorders. However, Itch deficiency in committed Treg cells did not alter in vitro suppressive activity as measured by coculture assay or the inhibitory capability of $\mathrm{CD}^{+} \mathrm{T}$ cell autoreactivity in the mouse adoptive transfer model of colitis. Many mechanisms have been proposed for the suppressive activity of Treg cells, including CTLA-4-mediated suppression of APCs, the competition for IL-2 consumption by the high-affinity IL-2 receptor $\alpha$ chain CD25, and the expression of IL-10 (reviewed in ref. 13). We indeed observed that the expression of various surface markers was not changed in Itch-/- $\mathrm{YFP}^{+}$Treg cells. In addition, the expression of IL-10, which has been shown to be critical for the maintenance of mucosal homeostasis and Treg function (30), is rather augmented in Itch ${ }^{-/-}$Treg cells, together with other Th2 cytokines. This may provide an explanation for the normal suppressive function of Itch ${ }^{-/}$Treg cells in the adoptive transfer colitis model.

An emerging concept of Treg cell regulation is the acquisition of other Th-specific transcription factors, which renders the Treg cells capable of specifically controlling the respective Th-mediated immune responses. Coexpression of the Th1 lineage transcription 

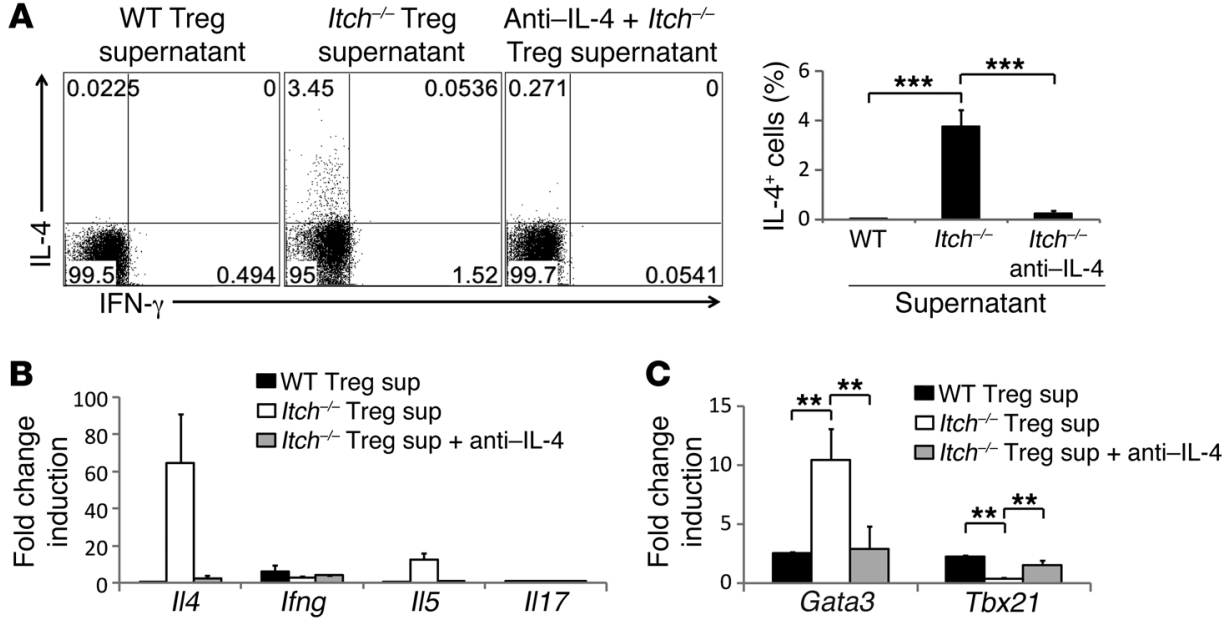

Figure 8

Itch-deficient Treg cells instruct the differentiation of naive T cells into Th2 in vitro. (A-C) FACS-sorted $\mathrm{CD}_{62} \mathrm{~L}^{\text {hi }} \mathrm{CD} 44^{\mathrm{l}}$ naive $\mathrm{CD} 4^{+} \mathrm{T}$ cells were cultured with supernatants (sup) from wild-type or Itch-deficient Treg cells for 5 days. Anti-IL-4 antibodies were added where indicated. (A) IL-4- and IFN- $\gamma-$ producing cells were analyzed by intracellular cytokine staining 5 hours after restimulation with anti-CD3/ CD28 antibodies. Data are representative of three independent experiments. (B and C) RT-PCR analysis of the indicated genes in these cells is shown. Data are compiled from three independent experiments. Error bars indicate the mean \pm SD. ${ }^{\star \star} P<0.01 ;{ }^{\star \star *} P<0.001$ (unpaired 2-tailed Student's $t$ test).

factor Tbx 21 is required for homeostasis and Treg cell function during a Th1 response (14), whereas the expression of STAT3 in Treg cells is required for the prevention of Th17 responses (15, 16). Of relevance to the current study, Treg cell-specific deletion of IRF4 results in excessive Th2 responses by the increased production of IL-4 and IL-5 cytokines (15). However, unlike Irf4-/- Treg cells, which displayed reduced IL-10 production and attenuated in vitro suppressive activity, Itch deficiency does not affect these events. It should be noted that Itch deficiency does not change Treg cell homeostasis or cell death. A recent study documented that Bcl-6 is required for the control of Th2 responses (36), since $B c l-6^{-/-}$Treg cells from conventional knockout mice displayed a phenotype similar to that in our current study. However, Itch deficiency does not affect Bcl-6 expression in an in vitro culture system (data not shown). The use of Treg cells from total Bcl-6-knockout mice, which display profound Th2 responses, may also complicate the interpretation. Thus, the present study suggests a unique pathway of molecular regulation by Itch that controls Th 2 responses independent of Foxp3 function.

The current study also addresses the controversial issue of Treg cell stability and maintenance under normal or inflammatory conditions. Previous studies have documented that Treg cells can lose their Foxp3 expression and be converted into pathogenic ex-Treg cells $(18,37)$. However, a study using acute and induced labeling of Foxp $3^{+}$Treg cells argued that they are very stable under various perturbed conditions (21). By using a newly generated fate-mapping strategy, Hori's group provided an alternative explanation in that the committed nTreg cells are stable, whereas a minor population of Treg cells displays transient or promiscuous Foxp3 expression and unstable properties (22). In our fate-mapping study using the double-reporter mice, we found that Itch deficiency does not affect the generation of the $\mathrm{RFP}^{+} \mathrm{YFP}^{-}$ex-Treg population, even in the 4-month-old mice with severe inflammation. In addition, in vitro culture of the $\mathrm{RFP}^{+} \mathrm{YFP}^{+}$Treg cells results in the genera- tion of $\mathrm{RFP}^{+} \mathrm{YFP}^{-}$ex-Treg cells, whose ratios are equivalent between control and Itch-/- Treg cells. Notably, Itch deficiency leads to a much higher production of Th2 cytokines by $\mathrm{RFP}^{+} \mathrm{YFP}^{-}$ex-Treg cells compared with $\mathrm{RFP}^{+} \mathrm{YFP}^{+}$Treg cells, whereas such cytokine production was minimal in both populations of the wild-type control mice. This suggests that Itch does not affect the stability of Treg cells per se. However, it converts Treg cells into Th2-like cells, which is further augmented by the loss of Foxp3 in ex-Treg cells. This implies that the regulation of Treg stability may not necessarily mean a loss of Foxp3 expression, but a functional modification of ex-Treg cells like the increased Th2-cytokine production, as revealed in the present study.

We propose that the Th2 cytokines produced by $\mathrm{Itch}^{-/-}$Treg cells account for the chronic inflammatory disorders and antigen-induced airway inflammation in $\mathrm{Itch}^{\mathrm{fl} / \mathrm{fl}} \mathrm{Foxp} 3^{\mathrm{Cre}}$ mice. Indeed, transferred Itch ${ }^{-/-}$Treg cells failed to suppress OVAinduced airway inflammation, but also exacerbated the inflammation. Our results suggest that $I_{t c h}{ }^{-/}$Treg cells produce sufficient amounts of Th2 cytokines to override the suppressive activity of these Tregs and to drive Th2 immune responses. We reason that IL-4 secreted from $\mathrm{Itch}^{-/-}$Treg cells may instruct naive T cells to develop into Th2 cells. Indeed, we observed that the culture supernatants from $\mathrm{Itch}^{-/-}$Treg cells drive the Th2 differentiation from naive $\mathrm{CD}^{+} \mathrm{T}$ cells. This result is consistent with the chronic activation and IL-4 production by Foxp3-negative CD $4^{+} \mathrm{T}$ cells in Itch $f^{f / f l}$ Foxp $3^{\mathrm{Cre}}$ mice. Another possibility is that $\mathrm{Itch}^{-/-}$Treg cells migrate to the inflammatory site and recruit innate effector cells that produce Th 2 cytokines. The recruited innate cells may play a role in the amplification of Th2 cytokines in Th2 responses. Interestingly, we observed a several-fold increase in Ccr4 mRNA expression in Itch-/Treg cells (data not shown), which is involved in the migration of Treg cells to the inflamed organ (38). However, other possible mechanisms, such as para-activated $\mathrm{CD}^{+} \mathrm{T}$ cells or other inflammatory cells, require further investigation. $\mathrm{Itch}^{-/-}$Treg cells showed increased expression of GATA3 and upregulated phosphorylation of STAT6, both of which have been implicated in IL-4 production $(39,40)$, even though an intrinsic role of STAT6 in Treg cells is questionable $(35,41)$. The effects of GATA3 and STAT 6 on IL-4 production were reversed by in vivo shRNA-based knockdown in $\mathrm{Itch}^{-/-}$Treg cells from bone marrow chimeric mice. An immediate question is whether phosphorylated STAT6 acts on the expression of the Gata3 gene or vice versa. It should be noted that previous studies documented that Itch targets JunB for ubiquitination and regulates JunB-mediated Il4 gene transcription (27, 29, 42). However, JunB is not expressed in resting Treg cells, and their stimulation does not lead to a further increase in JunB accumulation in $\mathrm{Itch}^{-/-}$Treg cells (data not shown). Thus, the present study raises a new question regarding the exact mechanism underlying Itch regulation of Th2 cytokines in Treg cells. At present, we consider it 
possible that Itch regulates the epigenetic program of Th2 cytokine gene transcription in Treg cells that tightly controls Th2 responses without affecting the suppressive activity and stability of these cells. Future mechanistic studies of these aspects may pave the way for therapeutic intervention in human allergic diseases.

\section{Methods}

Mice. C57BL/6, B6.SJL (C57BL/6 CD45.1+ congenic), and Rag1 $1 /$ mice were obtained from The Jackson Laboratory. The floxed Itch mice on a B6 background were generated by using a standard gene-targeting strategy with help from A. Tarakhvosky (Rockefeller University, New York, New York, USA). Foxp3-YFP-Cre mice were obtained from A. Rudensky (Memorial Sloan Kettering Cancer Center, New York, New York, USA). The fate-tracing ROSA26loxP-Stop-loxP RFP reporter mice were provided by H.J. Fehling (Institute of Immunology, University Clinics, Ulm, Germany). Foxp3-YFP-Cre mice were crossed with mice with a loxP-flanked Itch allele to produce Itch ${ }^{f / f l}$ Foxp3YFP-Cre (Itch ${ }^{l / f l}$ Foxp $\left.3^{\mathrm{Cre}}\right)$ mice. In some experiments, these mice were further crossed with ROSA26-loxP-Stop-loxP RFP reporter mice to generate WTR26R and Itch ${ }^{\mathrm{R} 26 \mathrm{R}}$ mice. All mice were housed in specific pathogen-free conditions.

Flow cytometry. Fluorescence-conjugated antibodies were purchased from BioLegend and eBioscience. Surface and intracellular staining was performed according to the manufacturer's protocol. For intracellular cytokine staining, cells isolated from various organs were stimulated with PMA $(100 \mathrm{ng} / \mathrm{ml})$ and ionomycin $(1 \mu \mathrm{g} / \mathrm{ml})$ in the presence of Golgi-stop (BD Biosciences) for 4 hours, and the fixed cells were incubated with the appropriate antibodies and analyzed by flow cytometry (BD Biosciences).

Plasmids. For constructing shRNA expression vectors, oligonucleotides were cloned into modified LMP vectors, which express the fluorescent protein mAmetrine1.1 (provided by S. Crotty, La Jolla Institute for Allergy and Immunology). Oligonucleotide sequences were as follows: STAT6 shRNA, 5'-TGCTGTTGACAGTGAGCGCTCAGATGCTTTCTGTTACAACTAGTGAAGCCACAGATGTAGTTGTAACAGAAAGCATCTGAATGCC TACTGCCTCGGA; and GATA3 shRNA, 5'-TGCTGTTGACAGTGAGCGAAAAGAGTGCCTCAAGTATCAGTAGTGAA-GCCACAGATGTACTGATACTTGAGGCACTCTTTCTGCCTACTGCCTCGGA-3'

In vitro suppression assay. Naive $\mathrm{CD} 4^{+} \mathrm{CD} 62 \mathrm{~L}^{+} \mathrm{CD} 25^{-} \mathrm{T}$ cells $\left(1 \times 10^{5}\right)$ were labeled with $5 \mu \mathrm{M}$ CellTrace violet dye (Invitrogen) for 10 minutes at $37^{\circ} \mathrm{C}$ in PBS and $0.1 \%$ BSA and were then cocultured with an increasing ratio of FACS-sorted Treg cells in the presence of $1 \mu \mathrm{g} / \mathrm{ml}$ anti-CD3 (BioLegend) plus irradiated splenocyte samples depleted of T cells $\left(5 \times 10^{4}\right)$. Anti-IL-10 antibody $(10 \mu \mathrm{g} / \mathrm{ml}$; BD Pharmingen) was added where indicated in Supplemental Figure 8. Four days later, cells were harvested, and violet dilution was measured by flow cytometric analysis.

Inflammatory scores. Histopathological scoring of tissue inflammation was carried out as previously described (43). Lung inflammation was scored separately for cellular infiltration around blood vessels and airways as follows: 0 , no infiltrates; 1 , few inflammatory cells; 2 , a ring of inflammatory cells 1 cell layer deep; 3 , a ring of inflammatory cells $2-4$ cells deep; 4 , a ring of inflammatory cells greater than 4 cells deep. A composite score was determined by adding the inflammatory scores for both vessels and airways. The severity of skin inflammation was scored from 0 to 4 : grade 0 , normal; grade 1 , hyperplasia of epidermis; and grades $2-4$, different amounts of infiltrating inflammatory cells in the skin.

Adoptive transfer model of colitis. Rag1-/- recipients were injected i.v. with FACS-sorted naive $\mathrm{CD} 4{ }^{+} \mathrm{CD} 25^{-} \mathrm{CD} 45 \mathrm{RB}^{\text {hi }} \mathrm{T}$ cells $\left(4 \times 10^{5}\right.$ cells/mouse $)$ from $\mathrm{CD} 45.1^{+}$congenic mice in the presence or absence of FACS-sorted $\mathrm{CD}^{+}{ }^{+} \mathrm{CD} 45 \mathrm{RB}^{\text {loYFPP }}{ }^{+}$Treg cells $\left(1 \times 10^{5}\right.$ cells/mouse $)$ from $\mathrm{Itch}^{+/}{ }^{+} \mathrm{Foxp} 3^{\mathrm{Cre}}$ or Itch ${ }^{f / f l} F o x p 3^{C r e}$ mice. Recipient Rag $1^{-/-}$mice were monitored weekly for 8 weeks. Histological assessment of colitis was performed by H\&E staining and analyzed by microscopy.
Retroviral transduction and bone marrow reconstitution. Phoenix-Eco packaging cells were used to generate shRNA-expressing bone marrow chimeric mice. Mature T cell-depleted bone marrow cells from Itchf/flf Foxp $3^{\mathrm{Cre}}$ mice were cultured for 24 hours in IL-3 (10 ng/ml), IL-6 (10 ng/ml), and SCF $(100 \mathrm{ng} / \mathrm{ml})$ (Peprotech) containing complete DMEM before the initial retroviral infection. The bone marrow cells were infected with retrovirus together with $5 \mu \mathrm{g} / \mathrm{ml}$ polybrene (Sigma-Aldrich) by "spin inoculation" ( $420 \mathrm{~g}$ for 1 hour). Two days after infection, retrovirally transduced bone marrow cells were injected into lethally irradiated ( $9 \mathrm{~Gy}$ ) CD $45.1^{+}$congenic recipient mice. Induction and assessment of EAE. Age- and sex-matched Itch ${ }^{+/+} F$ oxp $3^{3 r e}$ or Itch ${ }^{f / f l} \mathrm{Foxp} 3^{\mathrm{Cre}}$ mice were immunized s.c. with $100 \mu \mathrm{g}$ of a peptide consisting of amino acids 35-55 of MOG (AnaSpec). Immunization was performed by emulsifying the peptide with CFA (Difco Laboratories). Additionally, the mice received $100 \mathrm{ng}$ of pertussis toxin (List Biological Laboratories, Inc.) i.p. on days 0 and 2. Mice were monitored daily and scored for disease severity using the standard scale: 0 , no clinical signs; 1 , limp tail; 2 , paraperesis (partial hind limb paralysis); 3, paraplegia (complete hind limb paralysis); 4, paraplegia with forelimb weakness or paralysis; 5 , moribund or dead.

OVA-induced airway inflammation. Mice were immunized by i.p. injection of $20 \mu \mathrm{g}$ of OVA protein (Grade V; Sigma-Aldrich) adsorbed to $2 \mathrm{mg}$ of aluminum hydroxide (alum) gel (Imject Alum; Pierce Biotechnology) in PBS on days 0 and 14 . On days $25-27$, mice were challenged i.n. with OVA protein for 4 consecutive days. Mice were sacrificed 24 hours after the last OVA challenge and assessed for lung inflammation. For Treg cell transfer, wildtype $\mathrm{B} 6$ mice (recipients), as well as $\mathrm{Itch}{ }^{+/+} \mathrm{Foxp} 3^{\mathrm{Cre}}$ or $\mathrm{Itch} \mathrm{fl}^{\mathrm{fl} / \mathrm{F}} \mathrm{Foxp} 3^{\mathrm{Cre}}$ mice (Treg donors), were immunized by i.p. injection of $20 \mu \mathrm{g}$ of OVA protein adsorbed to alum on days 0 and 7 of the protocol (36). FACS-sorted $\mathrm{YFP}^{+}$ Treg cells $\left(3.5 \times 10^{5}\right)$ from OVA-sensitized $\mathrm{Itch}^{+/+} \mathrm{Foxp}^{\mathrm{Cre}}$ or Itch ${ }^{\text {flfl }} \mathrm{Foxp} 3^{\mathrm{Cre}}$ mice were injected into the sensitized wild-type B6 mice on day 14. One day after Treg transfer, recipient mice were challenged i.n. with OVA protein for 5 consecutive days. The collection of BAL fluid and determination of cytokine levels as well as histological analysis were performed.

Multicytokine assay. FACS-sorted $\mathrm{CD}^{+} \mathrm{RFP}^{+} \mathrm{YFP}^{+}$or $\mathrm{CD}^{+}{ }^{+} \mathrm{RFP}^{+} \mathrm{YFP}^{-}$ $T$ cells were stimulated with plate-coated anti-CD3 plus soluble anti-CD28 antibodies (Bio XCell) for 48 hours. Supernatants were collected, and the cytokines were detected using a multiplex cytokine kit from Bio-Rad according to the manufacturer's instructions.

In vitro Th2 cell differentiation. Wild-type naive $\mathrm{CD} 4^{+} \mathrm{T}$ cells $\left(3 \times 10^{5}\right.$ cells/ well) were cultured for 5 days on 96-well plates coated with anti-CD3/ CD28 antibodies in the presence or absence of supernatants from 2-day cultures of wild-type or Itch-deficient Treg cells $\left(2 \times 10^{5}\right.$ cells/well). AntiIL-4 antibody (10 $\mu \mathrm{g} / \mathrm{ml}$; BioLegend) was used to neutralize IL-4 activity.

Immunoblotting analysis. Cell pellets were lysed in $2 \times$ Laemmli sample buffer. Cell lysates were resolved by SDS-PAGE and immunoblotted with the appropriate antibodies. Antibodies against STAT6 and phospho-STAT6 (Tyr641) were purchased from Cell Signaling Technology. Anti-GATA3 and anti- $\beta$-actin were obtained from Santa Cruz Biotechnology Inc. AntiItch was from BD pharmingen.

Serum Ig ELISA. The concentrations of different Ig subclasses in mouse sera were analyzed using isotype-specific antibodies with a sandwich ELISA protocol. Monoclonal anti-mouse IgM, IgG1, IgG2a, IgG2b, IgG3, and IgA antibodies were from Sigma-Aldrich. Monoclonal anti-mouse IgE antibody was obtained from BD Pharmingen. ELISA was performed using biotinlabeled goat anti-mouse Ig (BD Pharmingen) and streptavidin-conjugated HRP (Southern Biotech).

Quantitative RT-PCR. RNA was isolated and CDNA was generated with a Superscript III kit (Invitrogen). Quantitative PCR was performed using SYBR Green Supermix (Bio-Rad).

Statistics. Data are shown as the mean \pm SD unless otherwise indicated in the legend. Student's $t$ tests and Kaplan-Meier survival analysis were per- 
formed using GraphPad Prism software (GraphPad Software Inc.). In all figures, ${ }^{*} P<0.05,{ }^{*} P<0.01$, and ${ }^{*}{ }^{*} P<0.001$.

Study approval. All animal experimental protocols were approved by members of the IACUC of the La Jolla Institute for Allergy and Immunology.

\section{Acknowledgments}

We thank A. Rudendsky and H.J. Fehling for providing their valuable mice and A. Tarakhvosky for help with generating Itch-floxed mice. K. Iwanami did the initial mouse characterization, and J.H. Lee and N. Xiao provided technical help. H.-S. Jin was supported in part by a National Research Foundation of Korea Grant funded by the Korean government (NRF-2009352-C00098). This work was supported by NIH-NIAID grants RO1AI62969 and RO1AI78272.

Received for publication February 13, 2013, and accepted in revised form August 1, 2013.

Address correspondence to: Yun-Cai Liu, Division of Cell Biology, La Jolla Institute for Allergy and Immunology, 9420 Athena Circle, La Jolla, California 92037, USA. Phone: 858.752.6810; Fax: 858.752.6986; E-mail: yuncail@liai.org.
1. Wing K, Sakaguchi S. Regulatory T cells exert checks and balances on self tolerance and autoimmunity. Nat Immunol. 2010;11(1):7-13.

2. Zheng Y, Rudensky AY. Foxp3 in control of the regulatory T cell lineage. Nat Immunol. 2007;8(5):457-462.

3. Hori S, Nomura T, Sakaguchi S. Control of regulatory $\mathrm{T}$ cell development by the transcription factor Foxp3. Science. 2003;299(5609):1057-1061.

4. Fontenot JD, Gavin MA, Rudensky AY. Foxp3 programs the development and function of CD $4^{+} \mathrm{CD} 25^{+}$ regulatory T cells. Nat Immunol. 2003;4(4):330-336.

5. Brunkow ME, et al. Disruption of a new forkhead/ winged-helix protein, scurfin, results in the fatal lymphoproliferative disorder of the scurfy mouse. Nat Genet. 2001;27(1):68-73.

6. Wildin RS, et al. X-linked neonatal diabetes mellitus, enteropathy and endocrinopathy syndrome is the human equivalent of mouse scurfy. Nat Genet. 2001;27(1):18-20.

7. Sakaguchi S, Yamaguchi T, Nomura T, Ono M. Regulatory $\mathrm{T}$ cells and immune tolerance. Cell. 2008;133(5):775-787.

8. Chen W, et al. Conversion of peripheral CD4 ${ }^{+} \mathrm{CD} 25^{-}$ naive $\mathrm{T}$ cells to $\mathrm{CD} 4^{+} \mathrm{CD} 25^{+}$regulatory $\mathrm{T}$ cells by TGF-beta induction of transcription factor Foxp3. J Exp Med. 2003;198(12):1875-1886.

9. Kretschmer K, Apostolou I, Hawiger D, Khazaie K, Nussenzweig MC, von Boehmer H. Inducing and expanding regulatory $\mathrm{T}$ cell populations by foreign antigen. Nat Immunol. 2005;6(12):1219-1227.

10. Floess $S$, et al. Epigenetic control of the foxp 3 locus in regulatory T cells. PLoS Biol. 2007;5(2):e38.

11. Yamaguchi T, Wing JB, Sakaguchi S. Two modes of immune suppression by Foxp $3\left(^{+}\right)$regulatory T cells under inflammatory or non-inflammatory conditions. Semin Immunol. 2011;23(6):424-430.

12. Shevach EM. Mechanisms of foxp $3^{+} \mathrm{T}$ regulatory cell-mediated suppression. Immunity. 2009; 30(5):636-645.

13. Wing JB, Sakaguchi S. Multiple treg suppressive modules and their adaptability. Front Immunol. 2012;3:178.

14. Koch MA, Tucker-Heard G, Perdue NR, Killebrew JR, Urdahl KB, Campbell DJ. The transcription factor T-bet controls regulatory $\mathrm{T}$ cell homeostasis and function during type 1 inflammation. Nat Immunol. 2009;10(6):595-602.

15. Zheng Y, et al. Regulatory T-cell suppressor program co-opts transcription factor IRF4 to control
$\mathrm{T}(\mathrm{H}) 2$ responses. Nature. 2009;458(7236):351-356. 16. Chaudhry A, et al. $\mathrm{CD}^{+}$regulatory $\mathrm{T}$ cells control TH17 responses in a Stat3-dependent manner. Science. 2009;326(5955):986-991.

17. Tsuji M, et al. Preferential generation of follicular $\mathrm{B}$ helper T cells from Foxp $3^{+} \mathrm{T}$ cells in gut Peyer's patches. Science. 2009;323(5920):1488-1492.

18. Zhou X, et al. Instability of the transcription factor Foxp3 leads to the generation of pathogenic memory T cells in vivo. Nat Immunol. 2009;10(9):1000-1007.

19. Takahashi R, et al. SOCS1 is essential for regulatory $\mathrm{T}$ cell functions by preventing loss of Foxp3 expression as well as IFN-\{gamma $\}$ and IL-17A production. J Exp Med. 2011;208(10):2055-2067.

20. Wang Y, Su MA, Wan YY. An essential role of the transcription factor GATA-3 for the function of regulatory T cells. Immunity. 2011;35(3):337-348.

21. Rubtsov YP, et al. Stability of the regulatory T cell lineage in vivo. Science. 2010;329(5999):1667-1671.

22. Miyao T, et al. Plasticity of Foxp3(+) T cells reflects promiscuous Foxp3 expression in conventional T cells but not reprogramming of regulatory $\mathrm{T}$ cells. Immunity. 2012;36(2):262-275.

23. Malynn BA, Ma A. Ubiquitin makes its mark on immune regulation. Immunity. 2010;33(6):843-852.

24. Bhoj VG, Chen ZJ. Ubiquitylation in innate and adaptive immunity. Nature. 2009;458(7237):430-437.

25. Kerscher O, Felberbaum R, Hochstrasser M. Modification of proteins by ubiquitin and ubiquitin-like proteins. Annu Rev Cell Dev Biol. 2006;22:159-180.

26. Liu YC. The E3 ubiquitin ligase Itch in T cell activation, differentiation, and tolerance. Semin Immunol. 2007;19(3):197-205.

27. Fang D, et al. Dysregulation of T lymphocyte function in itchy mice: a role for Itch in TH2 differentiation. Nat Immunol. 2002;3(3):281-287.

28. Venuprasad K, et al. The E3 ubiquitin ligase Itch regulates expression of transcription factor Foxp3 and airway inflammation by enhancing the function of transcription factor TIEG1. Nat Immunol. 2008;9(3):245-253.

29. Beal AM, Ramos-Hernandez N, Riling CR, Nowelsky EA, Oliver PM. TGF-beta induces the expression of the adaptor Ndfip1 to silence IL-4 production during iTreg cell differentiation. Nat Immunol. 2012;13(1):77-85.

30. Rubtsov YP, et al. Regulatory $\mathrm{T}$ cell-derived interleukin-10 limits inflammation at environmental interfaces. Immunity. 2008;28(4):546-558.
31. Luche H, Weber O, Nageswara Rao T, Blum C, Fehling HJ. Faithful activation of an extra-bright red fluorescent protein in "knock-in" Cre-reporter mice ideally suited for lineage tracing studies. EurJ Immunol. 2007;37(1):43-53.

32. Ouyang W, Beckett O, Ma Q, Li MO. Transforming growth factor-beta signaling curbs thymic negative selection promoting regulatory $\mathrm{T}$ cell development. Immunity. 2010;32(5):642-653.

33. Mantel PY, et al. GATA3-driven Th2 responses inhibit TGF-beta1-induced FOXP3 expression and the formation of regulatory T cells. PLoS Biol. 2007; 5(12):e329.

34. Wei J, Duramad O, Perng OA, Reiner SL, Liu YJ, Qin FX. Antagonistic nature of T helper $1 / 2$ developmental programs in opposing peripheral induction of Foxp $3^{+}$regulatory T cells. Proc Natl Acad Sci U S A. 2007;104(46):18169-18174.

35. Wohlfert EA, et al. GATA3 controls Foxp $3\left(^{+}\right)$regulatory $\mathrm{T}$ cell fate during inflammation in mice. J Clin Invest. 2011;121(11):4503-4515.

36. Sawant DV, et al. Bcl6 controls the th2 inflammatory activity of regulatory $T$ cells by repressing gata 3 function. J Immunol. 2012;189(10):4759-4769.

37. Murai $\mathrm{M}$, et al. Interleukin 10 acts on regulatory $\mathrm{T}$ cells to maintain expression of the transcription factor Foxp 3 and suppressive function in mice with colitis. Nat Immunol. 2009;10(11):1178-1184.

38. Faustino L, et al. Regulatory T cells migrate to airways via CCR4 and attenuate the severity of airway allergic inflammation. J Immunol. 2013;190(6):2614-2621.

39. Zheng W, Flavell RA. The transcription factor GATA-3 is necessary and sufficient for Th2 cytokine gene expression in CD4 T cells. Cell. 1997;89(4):587-596.

40. Zhu J, Guo L, Watson CJ, Hu-Li J, Paul WE. Stat6 is necessary and sufficient for IL-4's role in Th2 differentiation and cell expansion. J Immunol. 2001; 166(12):7276-7281.

41. Wang Y, Souabni A, Flavell RA, Wan YY. An intrinsic mechanism predisposes Foxp3-expressing regulatory $\mathrm{T}$ cells to Th2 conversion in vivo. J Immunol. 2010;185(10):5983-5992.

42. Gao M, et al. Jun turnover is controlled through JNK-dependent phosphorylation of the E3 ligase Itch. Science. 2004;306(5694):271-275.

43. Rivas MN, et al. MyD88 is critically involved in immune tolerance breakdown at environmental interfaces of Foxp3-deficient mice. J Clin Invest. 2012;122(5):1933-1947. 